\title{
Critical dynamics of the $O(n)$-symmetric relaxational models below the transition temperature
}

\author{
U. C. Täuber and F. Schwabl \\ Institut für Theoretische Physik, Physik-Department der Technischen Universität München, \\ James-Franck-Strasse, D-8046 Garching, Germany
}

(Received 17 December 1991)

\begin{abstract}
The critical dynamics of the $O(n)$-symmetric relaxational models with either nonconserved (model A) or conserved order parameter (model B) are studied below the transition temperature. As a consequence of Goldstone's theorem, the transverse modes are massless, implying infrared divergences in the theory along the entire coexistence curve. These Goldstone singularities can be treated within the fieldtheoretical formulation of the dynamical renormalization group by using the generalized regularization scheme as introduced by Amit and Goldschmidt, which has already been applied on the statics of the $\phi^{4}$ model below $T_{c}$ by Lawrie. We extend the formalism in several respects: (i) we generalize it to dynamical phenomena, (ii) taking advantage of the fact that the theory is exactly treatable in the coexistence limit, we do not use the $\epsilon$ expansion; (iii) the flow equations are solved numerically, thus allowing for a detailed description of the crossover from the critical isotropic Heisenberg fixed point to the infraredstable coexistence fixed point. We calculate the static susceptibilities as well as the dynamical correlation functions for models $\mathrm{A}$ and $\mathrm{B}$ within the complete crossover region, identifying the asymptotic coexistence anomalies and also a pronounced intermediate minimum of the effective critical exponents. Furthermore, the longitudinal dynamical correlation function $G_{L}(q, \omega)$ displays an anomalous line shape.
\end{abstract}

\section{INTRODUCTION}

This paper is concerned with the critical dynamics of he isotropic $n$-component time-dependent GinzburgJandau models $A$ and $B$ (according to the classification n Ref. 1) in the phase characterized by a spontaneously iroken global continuous symmetry. Their purely relaxaional behavior with either nonconserved (model A) or onserved (model B) order parameter constitutes the simlest conceivable model for dynamical phenomena in the icinity of a critical point. Above $T_{c}$, the features of nodel $A$ and $B$ have been extensively studied by Halpein, Hohenberg, and $\mathrm{Ma}^{2}$ using the dynamical renormalzation group and later also by De Dominicis, Brézin, and Jinn-Justin ${ }^{3}$ within a field-theoretical formulation. We hall apply the path-integral representation for the genralized Langevin equations as developed for dynamical ritical phenomena by Bausch, Janssen, and Wagner ${ }^{4}$ and )e Dominicis. ${ }^{5}$

With an $n$-component order parameter, the situation elow the transition temperature is to be treated sepaately, because parallel and perpendicular fluctuations vith respect to the spontaneous magnetization have to be istinguished, and generally two different length and time cales, characterized by the longitudinal and transverse orrelation lengths, appear. If the model displays a gloal continuous symmetry which is spontaneously broken elow $T_{c}$, the transverse correlation length diverges in he limit of zero external field. Although one of the quivalent directions in order-parameter space is selected, $o$ (free) energy is required for an infinitesimal quasistatic otation of the magnetization vector. This physical effect i mathematically expressed by the Goldstone theorem, ${ }^{6}$ stating that there is exactly one massless (bosonic) mode for each generator of the broken-symmetry subgroup. In the context of critical phenomena, massless modes can be traced back to a divergent length scale, which leads to characteristic infrared singularities in the theory. These are reflected in the nonanalytical behavior of appropriate correlation functions, the so-called coexistence anomalies in our case, and are, at least in principle, subject to experimental observation.

Therefore there has been considerable theoretical interest in the effect of Goldstone modes on the behavior of physical quantities in the low-temperature phase. Investigations by means of the renormalization group were performed by Brézin and Wallace, ${ }^{7}$ who were able to show that not only the transverse, but also the longitudinal static susceptibility $\chi_{L}$ diverges for vanishing external fields $h$ as

$$
\chi_{L} \propto h^{-\epsilon / 2},
$$

where

$$
0 \leq \epsilon=4-d<2
$$

parametrizes the space dimension $d$. In three dimensions this result has been obtained by Holstein and Primakoff, ${ }^{8}$ and it follows rigorously ${ }^{9}$ from Dyson's spin-wave theory, ${ }^{10}$ which becomes exact at low temperatures. Wallace and Zia calculated the equation of state, assuming that the transverse fluctuations behave canonically. ${ }^{11} \mathrm{~A}$ complete exponentiation of the Goldstone singularities was achieved by Nelson, ${ }^{12}$ who introduced a matching scheme by which a partial summation of the perturbation series is accomplished. In order to understand the coexistence behavior more deeply, Brézin and Zinn-Justin 
also investigated the nonlinear $\sigma$ model. ${ }^{13}$ They identified a new fixed point of Gaussian character governing the anomalies caused by the massless transverse modes.

The influence of Goldstone modes on the dynamics of the relaxational model $\mathbf{A}$ was examined in the limit $\mathbf{q} \rightarrow 0$ and $\omega \rightarrow 0$ by Mazenko using a $1 / n$ expansion. ${ }^{14}$ Further exponentiation schemes have been proposed by Schäfer and Horner ${ }^{15}$ for static phenomena, by Schäfer ${ }^{16}$ for dynamical phenomena, and also by Nicoll and Chang. ${ }^{17}$ Based on a general crossover theory by Amit and Goldschmidt, $^{18}$ Lawrie's work $^{19,20}$ provides a certain breakthrough, in the sense that his method leads to more transparent results than the previous attempts, it comprises exact statements in the regime dominated by the Goldstone modes alone, and it neatly fits in the standard field-theoretical formulation of the renormalization group.

Lawrie's idea was to change the point of view: not to concentrate on the fact of $n-1$ modes being massless, but rather to consider the freezing out of the longitudinal fluctuation. He realized the already implicitly stated similarity to the treatment of Amit and Goldschmidt's of bicritical points, ${ }^{18}$ where a crossover takes place from the critical behavior of an $m$-component subspace to the softening of all $n$ order-parameter fluctuations. In his papers Lawrie was able to reestablish and extend the former results. ${ }^{19,20}$ Although in principle the complete crossover region is accessible to the generalized renormalization scheme, however, he merely studied an approximate analytical solution of the asymptotic flow equations. We shall extend Lawrie's method to dynamical critical phenomena, but shall also go beyond his work by solving the renormalization-group equations to one-loop order numerically without making any use of further approximations. In particular, we shall refrain from the $\epsilon$ expansion, following the arguments by Schloms and Dohm, ${ }^{21}$ and especially exploiting the fact that the theory can be treated exactly in the Goldstone regime. Thereby the crossover itself can be thoroughly studied, and certain interesting features will be found.

In this paper we use the general theory in order to calculate the static and dynamical response functions. We remark, however, that also other correlation functions are within the scope of the formalism, e.g., the frequency-dependent specific heat, which constitutes the ultrasound attenuation coefficient (see Ref. 22). Although because of their neglecting of mode-coupling vertices the relaxational models are usually too crude to describe real systems, there are a few examples of normal-toincommensurate phase transitions whose dynamics fall into this universality class, e.g., the crystals of the general $A_{2} B X_{4}$ structure. As is shown in Ref. 22, soundattenuation measurements in these substances below $T_{c}$ can be very well explained by our theory. Perhaps even more important, because of the relative lucidity of Lawrie's approach (with our extensions), it is feasible to treat more complicated models, containing, for example, anisotropies or mode-coupling terms; this has not yet been possible with the former methods. We plan to come back to this topic in a further communication. The exact statements in the vicinity of the coexistence fixed point also provide an illuminating insight into the connections with the leading order of the $1 / n$ expansion, which has just recently been used to study dynamical coexistence anomalies. ${ }^{23}$

This paper is organized as follows: In the following section we introduce the time-dependent GinzburgLandau models $\mathrm{A}$ and $\mathrm{B}$ and give an outline to the general dynamical perturbation theory in the form proposed by Bausch, Janssen, and Wagner. ${ }^{4}$ In the third section we shall extend the general formalism to the phase with spontaneously broken symmetry. Special interest will be devoted to the so-called coexistence limit where the Goldstone modes dominate the entire physics. The connection to the $1 / n$ expansion will be established there, too. In the fourth section we shall proceed to discuss the specific modifications of the renormalization concepts which have to be introduced in order to describe the low-temperature phase correctly. Furthermore, we present the explicit one-loop results for the renormalization constants and flow functions of the relaxational models below $T_{c}$. The numerical solution of the flow equations will be extensively discussed and the scaling variable identified. In Sec. $\mathrm{V}$ we shall apply the formalism to the calculation of the static response and dynamical correlation functions for models $A$ and $B$ in the entire crossover region. Possibly observable effects will be emphasized. Finally, we shall discuss our results and provide an outlook on future developments. In the Appendix we list a number of important dynamical WardTakahashi identities and the diagrams and corresponding analytical results for the two-point vertex functions to one-loop order.

\section{MODEL}

\section{AND DYNAMICAL PERTURBATION THEORY}

In this section the $\mathrm{O}(n)$-symmetrical relaxational models will be introduced, and a short outline of the pathintegral formulation of dynamical perturbation theory of Bausch, Janssen, and Wagner ${ }^{4}$ will be presented. The specific features of the theory below $T_{c}$ will be described in Sec. III.

\section{A. Isotropic relaxational models A and B}

We are interested in the dynamical properties of materials undergoing a second-order phase transition which can be described by an $\mathrm{O}(n)$-symmetrical $\phi^{4}$ Hamiltonian

$$
\begin{aligned}
H\left[\left\{\phi_{0}^{\alpha}\right\}\right]=\int d^{d} x[ & \frac{r_{0}}{2} \sum_{\alpha=1}^{n} \phi_{0}^{\alpha}(\mathbf{x})^{2}+\frac{1}{2} \sum_{\alpha=1}^{n}\left[\nabla \phi_{0}^{\alpha}(\mathbf{x})\right]^{2} \\
& \left.+\frac{u_{0}}{4 !}\left[\sum_{\alpha=1}^{n} \phi_{0}^{\alpha}(\mathbf{x})^{2}\right]^{2}\right]
\end{aligned}
$$

The parameter $r_{0}$ is proportional to the distance from the mean-field critical temperature $T_{c}^{0}, r_{0} \propto T-T_{c}^{0}$, and hence comprises a shift $r_{0 c}$ of the transition temperature from $T_{c}^{0}$ to $T_{c}$ due to fluctuation effects and the reduced temperature variable with respect to $T_{c}$ : 


$$
r_{0}=r_{0 c}+\frac{T-T_{c}}{T}
$$

The positive coupling constant $u_{0}$ gives the strength of the anharmonicity within the order-parameter fluctuations described by an $n$-component field $\phi_{0}^{\alpha}, \alpha=1, \ldots, n$. In a state of thermal equilibrium, the Hamiltonian (2.1) defines the probability density for a specific configuration according to

$$
P\left[\left\{\phi_{0}^{\alpha}\right\}\right]=\frac{e^{-H\left[\left\{\phi_{0}^{\alpha}\right\}\right]}}{\int \mathcal{D}\left[\left\{\phi_{0}^{\alpha}\right\}\right] e^{-H\left[\left\{\phi_{0}^{\alpha}\right\}\right]}} .
$$

The critical dynamics of the system under consideration is then characterized by a set of generalized Langevin equations for the "slow" variables, which generally consist of (i) the order-parameter fluctuations (because of the critical "slowing-down" in the vicinity of a phase transition) and possibly (ii) conserved quantities that obey a continuity equation and therefore display propagating or diffusive hydrodynamical behavior. If the ordering field is the only relevant mode, the general structure of the equation of motion is

$$
\frac{\partial}{\partial t} \phi_{0}^{\alpha}(\mathbf{x}, t)=K_{0}^{\alpha}\left[\left\{\phi_{0}^{\alpha}\right\}\right](\mathbf{x}, t)+\zeta^{\alpha}(\mathbf{x}, t),
$$

where $K_{0}^{\alpha}\left[\left\{\phi_{0}^{\alpha}\right\}\right]$ contains the systematic part of the generalized forces, while the stochastic forces $\xi^{\alpha}$ subsume all the "fast" variables. In the simplest case, the dynamics is purely relaxational and no mode-coupling terms appear within $K_{0}^{\alpha}$ :

$K_{0}^{\alpha}\left[\left\{\phi_{0}^{\alpha}\right\}\right]=-\hat{L}_{0} \frac{\delta H\left[\left\{\phi_{0}^{\alpha}\right\}\right]}{\delta \phi_{0}^{\alpha}}=-\lambda_{0}(i \nabla)^{a} \frac{\delta H\left[\left\{\phi_{0}^{\alpha}\right\}\right]}{\delta \phi_{0}^{\alpha}}$.

Now two different situations have to be distinguished. Namely, either there is no conservation law for the order-parameter field, and the system just relaxes into the equilibrium state after a distortion [ $a=0$ in Eq. (2.4b)], or the order parameter is itself a conserved quantity and hence follows a diffusion equation $(a=2)$. According to the classification by Halperin and Hohenberg, ${ }^{1}$ Eqs. (2.4) together with (2.1) constitute the relaxational models A and $B$, respectively, for dynamical critical phenomena.

The probability distribution for the stochastic forces $\xi^{\alpha}$ is assumed to be Gaussian:

$W\left[\left\{\zeta^{\alpha}\right\}\right]$

$$
\propto \exp \left[-\frac{1}{4} \int d^{d} x \int d t \sum_{\alpha} \zeta^{\alpha}(\mathbf{x}, t)\left[\hat{L}_{0}^{-1} \zeta^{\alpha}(\mathbf{x}, t)\right]\right] .
$$

Consequently, the first two moments read

$$
\begin{aligned}
& \left\langle\zeta^{\alpha}(\mathbf{x}, t)\right\rangle=0, \\
& \left\langle\zeta^{\alpha}(\mathbf{x}, t) \xi^{\beta}\left(\mathbf{x}^{\prime}, t^{\prime}\right)\right\rangle=2 \hat{L}_{0} \delta^{\alpha \beta} \delta\left(\mathbf{x}-\mathbf{x}^{\prime}\right) \delta\left(t-t^{\prime}\right),
\end{aligned}
$$

and higher correlations either vanish or can be factorized into products of (2.6b). The numerical factor in (2.5) was precisely chosen in order to guarantee the Einstein relation (2.6b), which ensures that the equilibrium probability density is indeed given by the expression (2.3).

\section{B. Dynamical perturbation theory}

On the basis of Eq. (2.5), the path-integral formulation of dynamical perturbation theory can be developed according to Bausch, Janssen, Wagner ${ }^{4}$ and De Dominicis. ${ }^{5}$ At first, using the equation of motion (2.4a), the stochastic forces are eliminated, leading to a probability density for the order-parameter fluctuations themselves. However, the resulting Onsager-Machlup functional contains strong nonlinearities and also the inverse of the Onsager operator $\hat{L}_{0}$, which diverges in the case $a=2$. Therefore a Gaussian transformation that reduces the anharmonic terms by introducing purely auxiliary Martin-Siggia-Rose fields $\widetilde{\phi}_{0}^{\alpha}$ is most convenient. One eventually finds

$$
\begin{aligned}
P\left[\left\{\phi_{0}^{\alpha}\right\}\right] & =\int \mathscr{D}\left[\left\{i \widetilde{\phi}_{0}^{\alpha}\right\}\right] P\left[\left\{\widetilde{\phi}_{0}^{\alpha}\right\},\left\{\phi_{0}^{\alpha}\right\}\right] \\
& \propto \int \mathscr{D}\left[\left\{i \widetilde{\phi}_{0}^{\alpha}\right\}\right] e^{J\left[\left\{\phi_{0}^{\alpha}\right\},\left\{\phi_{0}^{\alpha}\right\}\right]},
\end{aligned}
$$

where the statistical weight for the configuration $\phi_{0}^{\alpha}$ is determined by the Janssen-De Dominicis functional

$$
J\left[\left\{\widetilde{\phi}_{0}^{\alpha}\right\},\left\{\phi_{0}^{\alpha}\right\}\right]=\int d^{d} x \int d t \sum_{\alpha}\left[\widetilde{\phi}_{0}^{\alpha} \hat{L}_{0} \widetilde{\phi}_{0}^{\alpha}-\widetilde{\phi}_{0}^{\alpha}\left(\frac{\partial \phi_{0}^{\alpha}}{\partial t}-K_{0}^{\alpha}\left[\left\{\phi_{0}^{\alpha}\right\}\right]\right)-\frac{1}{2} \frac{\delta K_{0}^{\alpha}}{\delta \phi_{0}^{\alpha}}\right] .
$$

Here the last term stems from the functional derivative originating in the change of variables from $\xi^{\alpha}$ to $\phi_{0}^{\alpha}$. However, as can be easily seen, it precisely cancels those contributions from the perturbation series which correspond to acausal diagrams and can therefore be dropped. ${ }^{4}$

We now split the dynamical functional (2.7b) into the harmonic part $J_{0}\left[\left\{\tilde{\phi}_{0}^{\alpha}\right\},\left\{\phi_{0}^{\alpha}\right\}\right]$ and the interaction $J_{\text {int }}\left[\left\{\tilde{\phi}_{0}^{\alpha}\right\},\left\{\phi_{0}^{\alpha}\right\}\right]$. For the time-dependent Ginzburg-Landau models $\mathbf{A}$ and $\mathbf{B}$, these are explicitly given by

$$
\begin{aligned}
& J_{0}\left[\left\{\widetilde{\phi}_{0}^{\alpha}\right\},\left\{\phi_{0}^{\alpha}\right\}\right]=\int_{q} \int_{\omega} \sum_{\alpha}\left\{\lambda_{0} q^{a} \widetilde{\phi}_{0}^{\alpha}(\mathbf{q}, \omega) \widetilde{\phi}_{0}^{\alpha}(-\mathbf{q},-\omega)-\widetilde{\phi}_{0}^{\alpha}(\mathbf{q}, \omega)\left[i \omega+\lambda_{0} q^{a}\left(r_{0}+q^{2}\right)\right] \phi_{0}^{\alpha}(-\mathbf{q},-\omega)\right\} \\
& J_{\mathrm{int}}\left[\left\{\widetilde{\phi}_{0}^{\alpha}\right\},\left\{\phi_{0}^{\alpha}\right\}\right]=-\frac{1}{6} \lambda_{0} u_{0} \int_{q_{1}, q_{2}, q_{3}, q_{4}} \int_{\omega_{1}, \omega_{2}, \omega_{3}, \omega_{4}} q_{1}^{a} \delta\left[\sum_{i} \mathbf{q}_{i}\right] \delta\left[\sum_{i} \omega_{i}\right] \sum_{\alpha, \beta} \widetilde{\phi}_{0}^{\alpha}\left(\mathbf{q}_{1}, \omega_{1}\right) \phi_{0}^{\alpha}\left(\mathbf{q}_{2}, \omega_{2}\right) \phi_{0}^{\beta}\left(\mathbf{q}_{3}, \omega_{3}\right) \phi_{0}^{\beta}\left(\mathbf{q}_{4}, \omega_{4}\right)
\end{aligned}
$$


here we have introduced the abbreviations

$$
\begin{aligned}
& \int_{q} \cdots=\frac{1}{(2 \pi)^{d}} \int d^{d} q \cdots, \\
& \int_{\omega} \cdots=\frac{1}{2 \pi} \int d \omega \cdots .
\end{aligned}
$$

The derivation of perturbation theory then follows the standard routes, and we shall give only a very brief sketch of what happens, mainly to introduce the definitions and quantities needed. Further details can be found in textbooks, e.g., in Ref. 24.

To start with, we define the generating functional

$$
Z\left[\left\{\widetilde{h}^{\alpha}\right\},\left\{h^{\alpha}\right\}\right] \propto \int \mathcal{D}\left[\left\{i \widetilde{\phi}_{0}^{\alpha}\right\}\right] \mathcal{D}\left[\left\{\phi_{0}^{\alpha}\right\}\right] \exp \left[J\left[\left\{\widetilde{\phi}_{0}^{\alpha}\right\},\left\{\phi_{0}^{\alpha}\right\}\right]+\int d^{d} x \int d t \sum_{\alpha}\left(\widetilde{h}^{\alpha} \widetilde{\phi}_{0}^{\alpha}+h^{\alpha} \phi_{0}^{\alpha}\right)\right],
$$

from which the $N$-point Green functions and cumulants are obtained by appropriate variational derivatives with respect to the sources $\widetilde{h}^{\alpha}$ and $h^{\alpha}$ :

$$
\begin{aligned}
G_{0\left\{\tilde{\phi}^{\left.\alpha_{i}\right\}\left\{\phi^{\alpha}\right\}}\right.}\left(\left\{\mathbf{x}_{i}, t_{i}\right\} ;\left\{\mathbf{x}_{j}, t_{j}\right\}\right) & =\left\langle\prod_{i, j} \widetilde{\phi}_{0}^{\alpha_{i}}\left(\mathbf{x}_{i}, t_{i}\right) \phi_{0}^{\alpha_{j}}\left(\mathbf{x}_{j}, t_{j}\right)\right\rangle \\
& =\left.\prod_{i, j} \frac{\delta}{\delta \widetilde{h}^{\alpha_{i}}\left(\mathbf{x}_{i}, t_{i}\right)} \frac{\delta}{\delta h^{\alpha}\left(\mathbf{x}_{j}, t_{j}\right)} Z\left[\left\{\widetilde{h}^{\alpha}\right\},\left\{h^{\alpha}\right\}\right]\right|_{\left\{\tilde{h}^{\alpha}\right\}=\left\{h^{\alpha}\right\}=0}, \\
G_{0\left\{\tilde{\phi}^{\left.\alpha_{i}\right\}\left\{\phi^{\alpha}\right\}}\right.}^{c}\left(\left\{\mathbf{x}_{i}, t_{i}\right\} ;\left\{\mathbf{x}_{j}, t_{j}\right\}\right) & =\left\langle\prod_{i, j} \widetilde{\phi}_{0}^{\alpha_{i}}\left(\mathbf{x}_{i}, t_{i}\right) \phi_{0}^{\alpha_{j}}\left(\mathbf{x}_{j}, t_{j}\right)\right\rangle^{c} \\
& =\left.\prod_{i, j} \frac{\delta}{\delta \tilde{h}^{\alpha_{i}}\left(\mathbf{x}_{i}, t_{i}\right)} \frac{\delta}{\delta h^{\alpha_{j}}\left(\mathbf{x}_{j}, t_{j}\right)} \ln Z\left[\left\{\widetilde{h}^{\alpha}\right\},\left\{h^{\alpha}\right\}\right]\right|_{\left\{\tilde{h}^{\alpha}\right\}=\left\{h^{\alpha}\right\}=0} .
\end{aligned}
$$

We remark that throughout this paper the index 0 denotes the unrenormalized, but interacting quantities, while their renormalized counterparts are written without indices. Inverting the matrix which couples the bilinear terms in the harmonic Janssen-De Dominicis functional (2.8a) yields the free two-point functions or propagators of the theory, as follows from the evaluation of the corresponding harmonic generating functional. One gets two different kinds of nonvanishing two-point functions, namely, the response propagators $G_{0 \tilde{\phi} \phi}^{c}(\mathbf{q}, \omega)$ and the correlation propagators $G_{0 \phi \phi}^{c}(\mathbf{q}, \omega)$. On the other hand, $(2.8 \mathrm{~b})$ provides the relaxational interaction vertex. The contribution of $v$ th order in the perturbation expansion for the $N$-point cumulants

$$
G_{0\left\{\tilde{\phi}^{\alpha}{ }^{\alpha}\right\}\left\{\phi^{\alpha j}\right\}}^{c}\left(\left\{\mathbf{x}_{i}, t_{i}\right\} ;\left\{\mathbf{x}_{j}, t_{j}\right\}\right)=\left\langle\prod_{i, j} \widetilde{\phi}_{0}^{\alpha_{i}}\left(\mathbf{x}_{i}, t_{i}\right) \phi_{0}^{\alpha_{j}}\left(\mathbf{x}_{j}, t_{j}\right) \sum_{v} \frac{1}{v !}\left(J_{\text {int }}\left[\left\{\widetilde{\phi}_{0}^{\alpha}\right\},\left\{\phi_{0}^{\alpha}\right\}\right]\right)^{v}\right\rangle_{0}^{c}
$$

is then represented by the connected Feynman graphs with $N$ external legs (propagators) and $v$ vertices.

We shall also need the so-called vertex functions. To arrive at their generating functional, one uses the fields

$$
\begin{aligned}
\widetilde{\phi}_{0}^{\alpha} & =\frac{\delta \ln Z\left[\left\{\widetilde{h}^{\alpha}\right\},\left\{h^{\alpha}\right\}\right]}{\delta \widetilde{h}^{\alpha}}, \\
\phi_{0}^{\alpha} & =\frac{\delta \ln Z\left[\left\{\widetilde{h}^{\alpha}\right\},\left\{h^{\alpha}\right\}\right]}{\delta h^{\alpha}}
\end{aligned}
$$

to perform a Legendre transformation

$$
\Gamma\left[\left\{\widetilde{\phi}_{0}^{\alpha}\right\},\left\{\phi_{0}^{\alpha}\right\}\right]=-\ln Z\left[\left\{\widetilde{h}^{\alpha}\right\},\left\{h^{\alpha}\right\}\right]+\int d^{d} x \int d t \sum_{\alpha}\left(\widetilde{h}^{\alpha} \widetilde{\phi}_{0}^{\alpha}+h^{\alpha} \phi_{0}^{\alpha}\right)
$$

The $N$-point vertex functions are then defined via

$$
\Gamma_{0\left\{\tilde{\phi}^{\alpha_{i}}\right\}\left\{\phi^{\alpha}\right\}}\left(\left\{\mathbf{x}_{i}, t_{i}\right\} ;\left\{\mathbf{x}_{j}, t_{j}\right\}\right)=\left.\prod_{i, j} \frac{\delta}{\delta \widetilde{\phi}_{0}^{\alpha_{i}}\left(\mathbf{x}_{i}, t_{i}\right)} \frac{\delta}{\delta \phi_{0}^{\alpha_{j}}\left(\mathbf{x}_{j}, t_{j}\right)} \Gamma\left[\left\{\widetilde{\phi}_{0}^{\alpha}\right\},\left\{\phi_{0}^{\alpha}\right\}\right]\right|_{\left\{\tilde{h}_{\alpha}\right\}=\left\{h^{\alpha}\right\}=0} .
$$


There is a simple connection between the two-point vertex functions and cumulants, ${ }^{4}$ namely,

$$
\begin{aligned}
& G_{0 \bar{\phi}^{\alpha} \phi^{\alpha}}^{c}(\mathbf{q}, \omega)=\frac{1}{\Gamma_{0 \bar{\phi}^{\alpha} \phi^{\alpha}}(-\mathbf{q},-\omega)}, \\
& G_{0 \phi^{\alpha} \phi^{\alpha}}^{c}(\mathbf{q}, \omega)=-\frac{\Gamma_{0 \bar{\phi}^{\alpha} \tilde{\phi}^{\alpha}}(\mathbf{q}, \omega)}{\left|\Gamma_{0 \tilde{\phi}^{\alpha} \phi^{\alpha}}(\mathbf{q}, \omega)\right|^{2}} .
\end{aligned}
$$

From these relations and the Dyson equation for the response propagators,

$$
G_{0 \tilde{\phi}^{\alpha} \phi^{\alpha}}^{c}(\mathbf{q}, \omega)^{-1}=G_{0 \tilde{\phi}^{\alpha} \phi^{\alpha}}^{c(0)}(\mathbf{q}, \omega)^{-1}-\Sigma_{0}^{\alpha}(\mathbf{q}, \omega),
$$

it becomes evident that the two-point vertex functions are graphically represented by the one-particle irreducible diagrams for the self-energy $\Sigma_{0}^{\alpha}$. [Note, however, the different signs in the arguments of Eqs. (2.16a) and (2.17).]

\section{Dynamical response and correlation functions}

To understand the physical meaning of the response propagators made up by one order parameter and one auxiliary field, let us add an additional term $-\Sigma_{\alpha} \widetilde{h}^{\alpha} \phi_{0}^{\alpha}$ to the Hamiltonian (2.1), corresponding to the application of an external field $\widetilde{h}^{\alpha}$. This leads to a modified Janssen-De Dominicis functional ${ }^{4}$

$$
J^{\tilde{h}}\left[\left\{\widetilde{\phi}_{0}^{\alpha}\right\},\left\{\phi_{0}^{\alpha}\right\}\right]=J\left[\left\{\widetilde{\phi}_{0}^{\alpha}\right\},\left\{\phi_{0}^{\alpha}\right\}\right]+\int d^{d} x \int d t \sum_{\alpha} \widetilde{h}^{\alpha} \hat{L}_{0} \widetilde{\phi}_{0}^{\alpha},
$$

from which we calculate the dynamical susceptibility

$$
\begin{aligned}
\chi_{0}^{\alpha \beta}\left(\mathbf{x}, t ; \mathbf{x}^{\prime}, t^{\prime}\right) & =\left.\frac{\delta\left\langle\phi_{0}^{\alpha}(\mathbf{x}, t)\right\rangle}{\delta \widetilde{h}^{\beta}\left(\mathbf{x}^{\prime}, t^{\prime}\right)}\right|_{\tilde{h}^{\beta}=0} \\
& =\left\langle\phi_{0}^{\alpha}(\mathbf{x}, t) \hat{L}_{0} \widetilde{\phi}_{0}^{\beta}\left(\mathbf{x}^{\prime}, t^{\prime}\right)\right\rangle
\end{aligned}
$$

and its Fourier transform

$$
\chi_{0}^{\alpha \beta}(\mathbf{q}, \omega)=\lambda_{0} q^{a} G_{0 \tilde{\phi}^{\alpha} \phi^{\beta}}(\mathbf{q}, \omega) .
$$

Hence the response propagators are intimately related to the dynamical response functions, while, on the other hand, the correlation propagators are identical with the correlation functions; these facts clearly explain the terminology.

From the causal properties of the response functions

$$
\begin{aligned}
& G_{0\left\{\tilde{\phi}^{\alpha_{i}}\right\}\left\{\phi^{\alpha_{j}}\right\}}^{c}\left(\left\{\mathbf{x}_{i}, t_{i}\right\} ;\left\{\mathbf{x}_{j}, t_{j}\right\}\right)=0 \text { if any } t_{i}>t_{j} \forall j, \\
& \Gamma_{0\left\{\bar{\phi}^{\left.\alpha_{i}\right\}\left\{\phi^{\alpha}\right\}}\right.}\left(\left\{\mathbf{x}_{i}, t_{i}\right\} ;\left\{\mathbf{x}_{j}, t_{j}\right\}\right)=0 \text { if any } t_{j}>t_{i} \forall i,
\end{aligned}
$$

and the behavior under time reversal, the following important fluctuation-dissipation theorem can be derived: ${ }^{14}$

$$
\chi_{0}^{\alpha \beta}\left(\mathbf{x}, t ; \mathbf{x}^{\prime}, t^{\prime}\right)=-\Theta\left(t-t^{\prime}\right) \frac{\partial}{\partial t}\left\langle\phi_{0}^{\alpha}(\mathbf{x}, t) \phi_{0}^{\beta}\left(\mathbf{x}^{\prime}, t^{\prime}\right)\right\rangle .
$$

Using the time-reversal invariance of the correlation function, one finds

$$
G_{0 \phi^{\alpha} \phi^{\beta}}(\mathbf{q}, \omega)=\frac{2 \lambda_{0} q^{a}}{\omega} \operatorname{Im} G_{0 \tilde{\phi}^{\alpha} \phi^{\beta}}(\mathbf{q}, \omega)
$$

Thus, in order to calculate the dynamic susceptibilities and correlation functions, we merely have to deal with the response propagators.

\section{SPONTANEOUSLY BROKEN SYMMETRY AND COEXISTENCE LIMIT}

We now come to the description of the phase with spontaneously broken symmetry. We start with the derivation of the Ward-Takahashi identity and then discuss the necessary modifications of the dynamical perturbation theory. Finally, we examine the so-called coexistence limit of the theory, which will turn out to be exactly treatable.

\section{A. Ward-Takahashi identity and Goldstone theorem}

Below $T_{c}$, a spontaneous magnetization appears, which we assume to point in the $n$th direction of the orderparameter space. It turns out to be convenient to introduce new fields according to $(\alpha=1, \ldots, n-1)$

$$
\left[\begin{array}{c}
\widetilde{\phi}_{0}^{\alpha} \\
\widetilde{\phi}_{0}^{n}
\end{array}\right]=\left(\begin{array}{l}
\widetilde{\pi}_{0}^{\alpha} \\
\widetilde{\sigma}_{0}
\end{array}\right), \quad\left(\begin{array}{l}
\phi_{0}^{\alpha} \\
\phi_{0}^{n}
\end{array}\right]=\left(\begin{array}{c}
\pi_{0}^{\alpha} \\
\sigma_{0}+\bar{\phi}_{0}
\end{array}\right),
$$

such that the longitudinal fluctuation also has zero expectation value,

$$
\left\langle\pi_{0}^{\alpha}\right\rangle=\left\langle\sigma_{0}\right\rangle=0 \text {. }
$$

In addition, we parametrize the order parameter according to

$$
\bar{\phi}_{0}=\left(\frac{3}{u_{0}}\right)^{1 / 2} m_{0},
$$

where $m_{0}$ has the dimension of a mass (inverse length).

The original dynamical functional (2.8) is invariant under rotations in the $n$-dimensional space of the orderparameter fluctuations $\phi_{0}^{\alpha}$ and simultaneously such transformations for the auxiliary fields $\widetilde{\phi}_{0}^{\alpha}$. Consequently, also the generating functional (2.10) displays an $\mathrm{O}(n)$ symmetry with respect to the source fields $h^{\alpha}$ and $\widetilde{h}^{\alpha}$. If we choose the transformation to affect the $\alpha$ and $n$ components, an infinitesimal rotation about an angle $\varepsilon$ has the form

$$
\left[\begin{array}{l}
\delta h^{\alpha} \\
\delta h^{n}
\end{array}\right)=\left(\begin{array}{l}
h^{\prime \alpha}-h^{\alpha} \\
h^{\prime n}-h^{n}
\end{array}\right)=\varepsilon\left(\begin{array}{c}
-h^{n} \\
h^{\alpha}
\end{array}\right)
$$

(and analogously for $\delta \widetilde{h}^{\alpha}, \delta \widetilde{h}^{n}$ ). Hence we have

$$
\begin{aligned}
\delta \ln Z=\varepsilon \int d^{d} x \int d t & {\left[\frac{\delta \ln Z}{\delta \widetilde{h}^{n}} \widetilde{h}^{\alpha}-\frac{\delta \ln Z}{\delta \widetilde{h}^{\alpha}} \widetilde{h}^{n}\right.} \\
& \left.+\frac{\delta \ln Z}{\delta h^{n}} h^{\alpha}-\frac{\delta \ln Z}{\delta h^{\alpha}} h^{n}\right]=0,
\end{aligned}
$$


which, via (2.14) and (2.15), can be rearranged into the Ward-Takahashi identity for the generating functional of the vertex functions:

$$
\int d^{d} x \int d t\left[\widetilde{\phi}_{0}^{n} \frac{\delta \Gamma}{\delta \widetilde{\phi}_{0}^{\alpha}}-\widetilde{\phi}_{0}^{\alpha} \frac{\delta \Gamma}{\delta \widetilde{\phi}_{0}^{n}}+\phi_{0}^{n} \frac{\delta \Gamma}{\delta \phi_{0}^{\alpha}}-\phi_{0}^{\alpha} \frac{\delta \Gamma}{\delta \phi_{0}^{n}}\right]=0
$$

The derivation of this dynamical Ward-Takahashi identity follows the same path as in the static case, of course (see Ref. 24).

In the low-temperature phase, characterized by a nonvanishing order parameter (3.1), and for a uniform and time-independent external field $\widetilde{h}^{n}$ [compare Eq. (2.18)], variation with respect to $\widetilde{\phi}_{0}^{n}$ and Fourier transformation yields

$$
\widetilde{\phi}_{0} \Gamma_{0 \tilde{\pi} \pi}(0,0)=\widetilde{h}^{n},
$$

where

$$
\Gamma_{0 \tilde{\pi}^{\alpha} \pi^{\beta}}(\mathbf{q}, \omega)=\Gamma_{0 \pi \pi}(\mathbf{q}, \omega) \delta^{\alpha \beta}
$$

defines the vertex functions without tensor indices. From Eq. (3.5) it follows that the transverse static susceptibility $G_{0 \tilde{\pi} \pi}^{c}(0,0)=\Gamma_{0 \pi \pi}(0,0)^{-1}$ diverges along the entire coexistence curve, i.e., for $T<T_{c}$ and vanishing external field $\widetilde{h}^{n}=0$. This is an example of the famous Goldstone theorem, ${ }^{6}$ and it implies that the $n-1$ transverse modes are massless. Hence the theory is critical in the sense that infrared divergences appear, leading to nonanalytical behavior of measurable correlation functions.

From the Ward-Takahashi identity (3.4b), a number of important exact relations between different vertex functions can be derived. Some of these which are relevant for the renormalization procedure are listed in Appendix A.

\section{B. Equation of state \\ and dynamical perturbation theory below $T_{c}$}

Inserting (3.1a) and (3.2) into (2.8) yields the dynamical functional for the ordered phase $J=J_{0}+J_{\text {int }}+J_{1}+$ const, with

$$
\begin{aligned}
& J_{0}\left[\left\{\widetilde{\pi}_{0}^{\alpha}\right\}, \widetilde{\sigma}_{0},\left\{\pi_{0}^{\alpha}\right\}, \sigma_{0}\right]=\int_{q} \int_{\omega}\left\{\sum_{\alpha} \lambda_{0} q^{a} \widetilde{\pi}_{0}^{\alpha}(\mathbf{q}, \omega) \widetilde{\pi}_{0}^{\alpha}(-\mathbf{q},-\omega)+\lambda_{0} q^{a} \widetilde{\sigma}_{0}(\mathbf{q}, \omega) \widetilde{\sigma}_{0}(-\mathbf{q},-\omega)\right. \\
& -\sum_{\alpha} \widetilde{\pi}_{0}^{\alpha}(\mathbf{q}, \omega)\left[i \omega+\lambda_{0} q^{a}\left[r_{0}+\frac{m_{0}^{2}}{2}+q^{2}\right]\right] \pi_{0}^{\alpha}(-\mathbf{q},-\omega) \\
& \left.-\widetilde{\sigma}_{0}(\mathbf{q}, \omega)\left[i \omega+\lambda_{0} q^{a}\left[r_{0}+\frac{3 m_{0}^{2}}{2}+q^{2}\right]\right] \sigma_{0}(-\mathbf{q},-\omega)\right\}, \\
& J_{\text {int }}\left[\left\{\widetilde{\pi}_{0}^{\alpha}\right\}, \widetilde{\sigma}_{0},\left\{\pi_{0}^{\alpha}\right\}, \sigma_{0}\right]=-\frac{1}{6} \lambda_{0} u_{0} \int_{q_{1}, q_{2}, q_{3}, q_{4}} \int_{\omega_{1}, \omega_{2}, \omega_{3}, \omega_{4}} q_{1}^{a} \delta\left(\sum_{i} \mathbf{q}_{i}\right] \delta\left[\sum_{i} \omega_{i}\right) \\
& \times\left[\sum_{\alpha, \beta} \widetilde{\pi}_{0}^{\alpha}\left(\mathbf{q}_{1}, \omega_{1}\right) \pi_{0}^{\alpha}\left(\mathbf{q}_{2}, \omega_{2}\right) \pi_{0}^{\beta}\left(\mathbf{q}_{3}, \omega_{3}\right) \pi_{0}^{\beta}\left(\mathbf{q}_{4}, \omega_{4}\right)\right. \\
& +\sum_{\alpha} \widetilde{\pi}_{0}^{\alpha}\left(\mathbf{q}_{1}, \omega_{1}\right) \pi_{0}^{\alpha}\left(\mathbf{q}_{2}, \omega_{2}\right) \sigma_{0}\left(\mathbf{q}_{3}, \omega_{3}\right) \sigma_{0}\left(\mathbf{q}_{4}, \omega_{4}\right) \\
& +\sum_{\alpha} \widetilde{\sigma}_{0}\left(\mathbf{q}_{1}, \omega_{1}\right) \pi_{0}^{\alpha}\left(\mathbf{q}_{2}, \omega_{2}\right) \pi_{0}^{\alpha}\left(\mathbf{q}_{3}, \omega_{3}\right) \sigma_{0}\left(\mathbf{q}_{4}, \omega_{4}\right) \\
& \left.+\widetilde{\sigma}_{0}\left(\mathbf{q}_{1}, \omega_{1}\right) \sigma_{0}\left(\mathbf{q}_{2}, \omega_{2}\right) \sigma_{0}\left(\mathbf{q}_{3}, \omega_{3}\right) \sigma_{0}\left(\mathbf{q}_{4}, \omega_{4}\right)\right] \\
& -\lambda_{0} \frac{\sqrt{3 u_{0}}}{6} m_{0} \int_{q_{1}, q_{2}, q_{3}} \int_{\omega_{1}, \omega_{2}, \omega_{3}} q_{1}^{a} \delta\left(\sum_{i} \mathbf{q}_{i}\right) \delta\left(\sum_{i} \omega_{i}\right) \\
& \times\left[\sum_{\alpha} 2 \widetilde{\pi}_{0}^{\alpha}\left(\mathbf{q}_{1}, \omega_{1}\right) \pi_{0}^{\alpha}\left(\mathbf{q}_{2}, \omega_{2}\right) \sigma_{0}\left(\mathbf{q}_{3}, \omega_{3}\right)\right. \\
& \left.+\sum_{\alpha} \widetilde{\boldsymbol{\sigma}}_{0}\left(\mathbf{q}_{1}, \omega_{1}\right) \pi_{0}^{\alpha}\left(\mathbf{q}_{2}, \omega_{2}\right) \pi_{0}^{\alpha}\left(\mathbf{q}_{3}, \omega_{3}\right)+3 \widetilde{\sigma}_{0}\left(\mathbf{q}_{1}, \omega_{1}\right) \sigma_{0}\left(\mathbf{q}_{2}, \omega_{2}\right) \sigma_{0}\left(\mathbf{q}_{3}, \omega_{3}\right)\right],
\end{aligned}
$$


and

$$
\begin{aligned}
J_{1}\left[\widetilde{\sigma}_{0}\right]=- & \lambda_{0}\left(\frac{3}{u_{0}}\right)^{1 / 2} m_{0}\left(r_{0}+\frac{m_{0}^{2}}{2}\right) \\
& \times \int_{q} \int_{\omega} q^{a} \widetilde{\sigma}_{0}(-\mathbf{q},-\omega) .
\end{aligned}
$$

By evaluation of the condition $\left\langle\sigma_{0}\right\rangle=0$, we can eliminate the temperature variable $r_{0}$ in favor of the mass parameter $m_{0}$. Within the first order of perturbation theory, only the Hartree loops are to be taken into account, and one arrives at

$$
\begin{aligned}
r_{0}+ & \frac{m_{0}^{2}}{2}=A, \\
A= & -\frac{n-1}{6} u_{0} \int_{k} \frac{1}{r_{0}+m_{0}^{2} / 2+k^{2}} \\
& -\frac{1}{2} u_{0} \int_{k} \frac{1}{r_{0}+3 m_{0}^{2} / 2+k^{2}} .
\end{aligned}
$$

Of course, we could have derived the same result from the original static functional (2.1) (see Ref. 19). The integrals in (3.8b) are ultraviolet divergent when the cutoff in momentum space is extended to infinity. This unphysical singularity can be cured by a regularization procedure. Using the particularly convenient dimensional regularization scheme invented by 't Hooft and Velt$\operatorname{man}^{25}$ and introduced into the theory of critical phenomena by Lawrie, ${ }^{26}$ the counterterm $A$ explicitly reads

$$
\begin{aligned}
A= & \frac{n-1}{6 \epsilon} u_{0} A_{d}\left(r_{0}+\frac{m_{0}^{2}}{2}\right]^{1-\epsilon / 2} \\
& +\frac{1}{2 \epsilon} u_{0} A_{d}\left(r_{0}+\frac{3 m_{0}^{2}}{2}\right)^{1-\epsilon / 2},
\end{aligned}
$$

where $A_{d}$ is a geometrical factor related to the surface of the $d$-dimensional unit sphere (see Ref. 21):

$$
A_{d}=S_{d} \Gamma\left(3-\frac{d}{2}\right) \Gamma\left(\frac{d}{2}-1\right)=\frac{\Gamma(3-d / 2)}{2^{d-2} \pi^{d / 2}(d-2)} .
$$

Note that the expression (3.8c) is singular both at four $(\epsilon=0)$ and two $(\epsilon=2)$ dimensions, which is the formal origin of the restriction (1.2). Physically, for $d \geq 4$, because of the Ginzburg-Levanyuk criterion, fluctuation effects are negligible, and the theory is essentially Gaussian, with logarithmic corrections at the upper critical dimension $d_{c}=4$. At two dimensions the Mermin-Wagner theorem ${ }^{27,28}$ states that there is no long-range order for a system with a continuous global symmetry and shortrange interaction. Therefore the really interesting case for the study of coexistence anomalies is in fact $d=3$ $(\epsilon=1)$.
It is important to realize that by using the variable $m_{0}$ instead of $r_{0}$ we have already taken account of the $T_{c}$ shift $r_{0 c}$. Indeed, for $m_{0}=0$ we must have $r_{0}=r_{0 c}$, which with the help of (3.8c) allows us to determine the $T_{c}$ shift to lowest order:

$$
r_{0 c}=\left(\frac{n+2}{6 \epsilon} u_{0} A_{d}\right)^{2 / \epsilon} .
$$

Schloms and Dohm ${ }^{21}$ have emphasized that $r_{0 c}$ does not vanish in the dimensional regularization scheme if $\epsilon$ expansion is not applied. Furthermore, $r_{0 c}$ is obviously a nonanalytical function of the coupling constant $u_{0}{ }^{29}$ If we do not want to use the $\epsilon$ expansion, it is therefore essential to use a temperature variable which already includes the $T_{c}$ shift properly. In the ordered phase, certainly $m_{0}$ is a choice near at hand.

Using (3.8a), the Janssen-De Dominicis functional becomes $J=J_{0}+J_{\text {int }}+J_{\mathrm{CT}}$, where the harmonic part now reads

(a) Propagators:
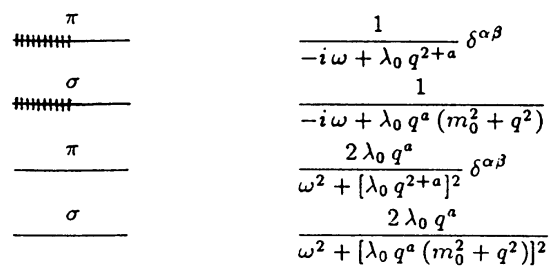

(b) Vertices:

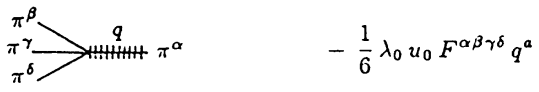

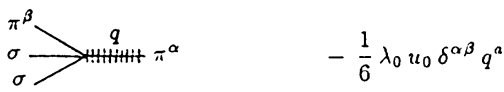

$$
\begin{aligned}
& \pi_{\sigma}^{\pi^{\alpha}}>\stackrel{q}{q} \sigma \quad-\frac{1}{6} \lambda_{0} u_{0} \delta^{\alpha \beta} q^{\alpha} \\
& { }_{\sigma}^{\sigma}{ }_{\sigma}>\stackrel{q}{\sigma} \quad-\frac{1}{6} \lambda_{0} u_{0} q^{a} \\
& { }_{\sigma}^{\beta} q^{q} \quad-\lambda_{0} \frac{\sqrt{3 u_{0}}}{3} m_{0} \delta^{\alpha \beta} q^{a} \\
& \pi^{\beta}>\stackrel{q}{q} \sigma \quad-\lambda_{0} \frac{\sqrt{3 u_{0}}}{6} m_{0} \delta^{\alpha \beta} q^{a} \\
& { }_{\sigma}^{\sigma}{ }_{\sigma}^{q} \sigma+\lambda_{0} \frac{\sqrt{3 u_{0}}}{2} m_{0} q^{a}
\end{aligned}
$$

(c) Counterterm:

$$
\times \quad A=-\frac{n-1}{6} u_{0} \int_{k} \frac{1}{k^{2}}-\frac{1}{2} u_{0} \int_{k} \frac{1}{m_{0}^{2}+k^{2}}
$$

FIG. 1. Basic elements of the dynamical perturbation theory for the relaxational models below $T_{c}$. 


$$
\begin{aligned}
J_{0}\left[\left\{\widetilde{\pi}_{0}^{\alpha}\right\}, \widetilde{\sigma}_{0},\left\{\pi_{0}^{\alpha}\right\}, \sigma_{0}\right]=\int_{q} \int_{\omega}[ & \sum_{\alpha} \lambda_{0} q^{a} \widetilde{\pi}_{0}^{\alpha}(\mathbf{q}, \omega) \widetilde{\pi}_{0}^{\alpha}(-\mathbf{q},-\omega)+\lambda_{0} q^{a} \widetilde{\sigma}_{0}(\mathbf{q}, \omega) \widetilde{\sigma}_{0}(-\mathbf{q},-\omega) \\
& -\sum_{\alpha} \widetilde{\pi}_{0}^{\alpha}(\mathbf{q}, \omega)\left[i \omega+\lambda_{0} q^{2+a}\right] \pi_{0}^{\alpha}(-\mathbf{q},-\omega) \\
& \left.-\widetilde{\sigma}_{0}(\mathbf{q}, \omega)\left[i \omega+\lambda_{0} q^{a}\left(m_{0}^{2}+q^{2}\right)\right] \sigma_{0}(-\mathbf{q},-\omega)\right],
\end{aligned}
$$

clearly displaying that the transverse mass vanishes. From (3.11a) we can then determine the four propagators of our theory, while the four- and three-vertices can be read off from the interaction (3.7b). The one-vertex (3.7c) is replaced by the counterterm

$$
\begin{aligned}
J_{\mathrm{CT}}\left[\left\{\widetilde{\pi}_{0}^{\alpha}\right\}, \widetilde{\sigma}_{0},\left\{\pi_{0}^{\alpha}\right\}, \sigma_{0}\right]= & -\lambda_{0} A \int_{q} \int_{\omega} q^{a}\left[\sum_{\alpha} \widetilde{\pi}_{0}^{\alpha}(\mathbf{q}, \omega) \pi_{0}^{\alpha}(-\mathbf{q},-\omega)+\widetilde{\sigma}_{0}(\mathbf{q}, \omega) \sigma_{0}(-\mathbf{q},-\omega)\right] \\
& -\lambda_{0}\left(\frac{3}{u_{0}}\right)^{1 / 2} m_{0} A \int_{q} \int_{\omega} q^{a} \widetilde{\sigma}_{0}(-\mathbf{q},-\omega) .
\end{aligned}
$$

The basic ingredients of the dynamical perturbation theory below $T_{c}$, as well as the corresponding graphical representations, are listed in Fig. 1.

\section{Properties of the coexistence limit}

The theory at the critical temperature $T_{c}$ is recovered from (3.11) and (3.7b) by setting $m_{0}=0$, and in particular the three-vertices disappear. We are, however, especially interested in the opposite limit $m_{0} \rightarrow \infty$, which, according to (3.8a) and (2.2), corresponds to $T=0$. This is because the renormalization-group analysis of Sec. IV will show that the renormalized counterpart of the mass parameter $m$ indeed flows to infinity when momentum and frequency tend to zero. Physically, this simply describes the "freezing out" of the longitudinal mode, whose fluctuations eventually become negligible, while the transverse modes stay massless. In the case of "maximal" symmetry breaking, the $n-1$ Goldstone modes will prevail. It may therefore be expected that this so-called coexistence limit can be simulated by letting the number of components $n$ tend to infinity, in which case the theory is tractable within the leading order of the $1 / n$ expansion.

In order to come to a more accurate notion of the coexistence limit, we devote this subsection to the examination of the unrenormalized model for the special case $m_{0} \rightarrow \infty$. As stated above, the relevance of this parameter study should become obvious in the following section. For convenience we write ${ }^{19}$

$$
\widetilde{s}_{0}=m_{0} \widetilde{\sigma}_{0}, \quad s_{0}=m_{0} \sigma_{0},
$$

and take the limit $m_{0} \rightarrow \infty$ in the dynamical functionals (3.11) and (3.7b), assuming that $u_{0} / m_{0}^{2} \rightarrow 0$. Only a few terms survive this procedure, namely,

$$
\begin{aligned}
& J_{\infty}\left[\left\{\widetilde{\pi}_{0}^{\alpha}\right\}, \widetilde{s}_{0},\left\{\pi_{0}^{\alpha}\right\}, s_{0}\right]=\int_{q} \int_{\omega}\left[\sum_{\alpha} \lambda_{0} q^{a} \widetilde{\pi}_{0}^{\alpha}(\mathbf{q}, \omega) \widetilde{\pi}_{0}^{\alpha}(-\mathbf{q},-\omega)\right.
\end{aligned}
$$

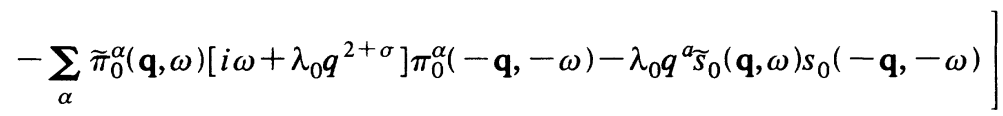

$$
\begin{aligned}
& -\frac{1}{6} \lambda_{0} u_{0} \int_{q_{1}, q_{2}, q_{3}, q_{4}} \int_{\omega_{1}, \omega_{2}, \omega_{3}, \omega_{4}} q_{1}^{a} \delta\left(\sum_{i} \mathbf{q}_{i}\right) \delta\left(\sum_{i} \omega_{i}\right) \\
& \times \sum_{\alpha, \beta} \widetilde{\pi}_{0}^{\alpha}\left(\mathbf{q}_{1}, \omega_{1}\right) \pi_{0}^{\alpha}\left(\mathbf{q}_{2}, \omega_{2}\right) \pi_{0}^{\beta}\left(\mathbf{q}_{3}, \omega_{3}\right) \pi_{0}^{\beta}\left(\mathbf{q}_{4}, \omega_{4}\right) \\
& -\lambda_{0} \frac{\sqrt{3 u_{0}}}{6} \int_{q_{1}, q_{2}, q_{3}} \int_{\omega_{1}, \omega_{2}, \omega_{3}} q^{a} \delta\left(\sum_{i} \mathbf{q}_{i}\right) \delta\left(\sum_{i} \omega_{i}\right) \\
& \times\left[\sum_{\alpha} 2 \widetilde{\pi}_{0}^{\alpha}\left(\mathbf{q}_{1}, \omega_{1}\right) \pi_{0}^{\alpha}\left(\mathbf{q}_{2}, \omega_{2}\right) s_{0}\left(\mathbf{q}_{3}, \omega_{3}\right)+\sum_{\alpha} \widetilde{s}_{0}\left(\mathbf{q}_{1}, \omega_{1}\right) \pi_{0}^{\alpha}\left(\mathbf{q}_{2}, \omega_{2}\right) \pi_{0}^{\alpha}\left(\mathbf{q}_{3}, \omega_{3}\right)\right] \\
& -\lambda_{0} A \int_{q} \int_{\omega} q_{1}^{a} \sum_{\alpha} \widetilde{\pi}_{0}^{\alpha}(\mathbf{q}, \omega) \pi_{0}^{\alpha}(-\mathbf{q},-\omega)-\lambda_{0}\left(\frac{3}{u_{0}}\right)^{1 / 2} A \int_{q} \int_{\omega} q^{a} \widetilde{\sigma}_{0}(-\mathbf{q},-\omega) .
\end{aligned}
$$

Lawrie was able to show that by introducing a new longitudinal field the static functional could be mapped onto a Gaussian Hamiltonian. ${ }^{19}$ Because the relaxational vertex is essentially of static origin, we expect an analogous situation here. In fact, if we set ${ }^{30}$ 


$$
\varphi_{0}(\mathbf{q}, \omega)=s_{0}(\mathbf{q}, \omega)+\frac{\sqrt{3 u_{0}}}{6} \int_{q^{\prime}} \int_{\omega^{\prime}} \sum_{\alpha=1}^{n-1} \pi_{0}^{\alpha}\left(\mathbf{q}^{\prime}, \omega^{\prime}\right) \pi_{0}^{\alpha}\left(\mathbf{q}-\mathbf{q}^{\prime}, \omega-\omega^{\prime}\right)+\left(\frac{3}{u_{0}}\right)^{1 / 2} A \delta(\mathbf{q}) \delta(\omega)
$$

and, similarly for the Martin-Siggia-Rose auxiliary fields (with $q \neq 0$ in case of a conserved order parameter),

$$
\widetilde{\varphi}_{0}(\mathbf{q}, \omega)=\widetilde{s}_{0}(\mathbf{q}, \omega)+\frac{\sqrt{3 u_{0}}}{3} \int_{q^{\prime}} \int_{\omega^{\prime}} \frac{q^{\prime a}}{q^{a}} \sum_{\alpha=1}^{n-1} \widetilde{\pi}_{0}^{\alpha}\left(\mathbf{q}^{\prime}, \omega^{\prime}\right) \pi_{0}^{\alpha}\left(\mathbf{q}-\mathbf{q}^{\prime}, \omega-\omega^{\prime}\right),
$$

then the asymptotic Janssen-De Dominicis functional

$$
\begin{aligned}
J_{\infty}\left[\left\{\widetilde{\pi}_{0}^{\alpha}\right\}, \widetilde{\varphi}_{0},\left\{\pi_{0}^{\alpha}\right\}, \varphi_{0}\right]=\int_{q} \int_{\omega}[ & \sum_{\alpha} \lambda_{0} q^{a} \widetilde{\pi}_{0}^{\alpha}(\mathbf{q}, \omega) \widetilde{\pi}_{0}^{\alpha}(-\mathbf{q},-\omega) \\
& \left.-\sum_{\alpha} \widetilde{\pi}_{0}^{\alpha}(\mathbf{q}, \omega)\left[i \omega+\lambda_{0} q^{2+a}\right] \pi_{0}^{\alpha}(-\mathbf{q},-\omega)-\lambda_{0} q^{a} \widetilde{\varphi}_{0}(\mathbf{q}, \omega) \varphi_{0}(-\mathbf{q},-\omega)\right]
\end{aligned}
$$

becomes harmonic in these fields. The longitudinal mode has ceased to fluctuate at all, and only the Goldstone modes persist. The transverse propagators are exactly those from zero-order perturbation theory,

$$
\begin{aligned}
& G_{0 \pi^{\alpha} \pi^{\beta}}^{\infty}(\mathbf{q}, \omega)=\frac{1}{-i \omega+\lambda_{0} q^{2+a}} \delta^{\alpha \beta}, \\
& G_{0 \pi^{\alpha} \pi^{\beta}}^{\infty}(\mathbf{q}, \omega)=\frac{2 \lambda_{0} q^{a}}{\omega^{2}+\left[\lambda_{0} q^{2+a}\right]^{2}} \delta^{\alpha \beta} ;
\end{aligned}
$$

hence the Goldstone modes behave canonically, as has been anticipated by Brézin and Wallace ${ }^{7}$ and also assumed by Wallace and $\mathrm{Zia}^{11}$ and Mazenko. ${ }^{14}$ We can already conclude that the field renormalizations for the transverse fluctuations as well as the renormalization for the time scale $\lambda_{0}$ disappear in the limit $m_{0} \rightarrow \infty$.

Using the definitions (3.12) and (3.14), we conclude

$$
\begin{aligned}
\left\langle\widetilde{\sigma}_{0} \sigma_{0}\right\rangle=\frac{1}{m_{0}^{2}} & \left\langle\widetilde{\varphi}_{0} \varphi_{0}\right\rangle-\frac{\sqrt{3 u_{0}}}{3} \sum_{\alpha}\left\langle\left[\widetilde{\pi}_{0}^{\alpha} \pi_{0}^{\alpha}\right] \varphi_{0}\right\rangle-\frac{\sqrt{3 u_{0}}}{6} \sum_{\alpha}\left\langle\widetilde{\varphi}_{0}\left[\pi_{0}^{\alpha} \pi_{0}^{\alpha}\right]\right\rangle \\
& \left.+\frac{u_{0}}{6} \sum_{\alpha, \beta}\left\langle\left[\widetilde{\pi}_{0}^{\alpha} \pi_{0}^{\alpha}\right]\left[\pi_{0}^{\beta} \pi_{0}^{\beta}\right]\right\rangle-\left(\frac{3}{u_{0}}\right)^{1 / 2} A\left\langle\widetilde{\varphi}_{0}\right\rangle+A \sum_{\alpha}\left\langle\left[\widetilde{\pi}_{0}^{\alpha} \pi_{0}^{\alpha}\right]\right\rangle\right], \\
\left\langle\sigma_{0} \sigma_{0}\right\rangle=\frac{1}{m_{0}^{2}}[ & \left\langle\varphi_{0} \varphi_{0}\right\rangle-\frac{\sqrt{3 u_{0}}}{3} \sum_{\alpha}\left\langle\left[\pi_{0}^{\alpha} \pi_{0}^{\alpha}\right] \varphi_{0}\right\rangle \\
& \left.+\frac{u_{0}}{12} \sum_{\alpha, \beta}\left\langle\left[\pi_{0}^{\alpha} \pi_{0}^{\alpha}\right]\left[\pi_{0}^{\beta} \pi_{0}^{\beta}\right]\right\rangle-2\left(\frac{3}{u_{0}}\right)^{1 / 2} A\left\langle\varphi_{0}\right\rangle+A \sum_{\alpha}\left\langle\left[\pi_{0}^{\alpha} \pi_{0}^{\alpha}\right]\right\rangle\right],
\end{aligned}
$$

where the symbolic notations $[\pi \pi]$ and $[\tilde{\pi} \pi]$ stand for the transverse composite operators defined by the integrals on the right-hand side (RHS) of (3.14). With the properties of the asymptotic dynamical functional, we finally get, for the longitudinal correlation functions in the coexistence limit,

$$
\begin{aligned}
& G_{0 \sigma \sigma}^{\infty}(\mathbf{q}, \omega)=\frac{1}{\lambda_{0} m_{0}^{2} q^{a}}\left[1+\frac{n-1}{3} u_{0} \int_{k} \frac{(\mathbf{q} / 2+\mathbf{k})^{a}}{(\mathbf{q} / 2-\mathbf{k})^{2}} \frac{1}{-i \omega / \lambda_{0}+(\mathbf{q} / 2+\mathbf{k})^{2+a}+(\mathbf{q} / 2-\mathbf{k})^{2+a}}\right), \\
& G_{0 \sigma \sigma}^{\infty}(\mathbf{q}, \omega)=\frac{1}{\lambda_{0} m_{0}^{2}} \frac{n-1}{3} u_{0} \operatorname{Re} \int_{k} \frac{1}{(\mathbf{q} / 2+\mathbf{k})^{2}(\mathbf{q} / 2-\mathbf{k})^{2}} \frac{1}{-i \omega / \lambda_{0}+(\mathbf{q} / 2+\mathbf{k})^{2+a}+(\mathbf{q} / 2-\mathbf{k})^{2+a}} .
\end{aligned}
$$

These exact results coincide with the one-loop expressions for the longitudinal functions in the limit $m_{0} \rightarrow \infty$ (compare Appendix B). Noting that the one-loop contributions for the transverse functions vanish for large $m_{0}$, we have established the important fact that in the coexistence limit the one-loop theory for the two-point cumulants becomes exact.
With the aid of (2.16), we can deduce another interesting statement for the two-point vertex functions. By inspection of $(3.17 \mathrm{a})$ it is evident that they are given by the geometric series of transverse loops depicted in Fig. 2. Now these diagrams are precisely those that have to be summed up within the leading order of the $1 / n$ expansion. $^{23,30}$ Therefore the coexistence limit for the two- 
(a)

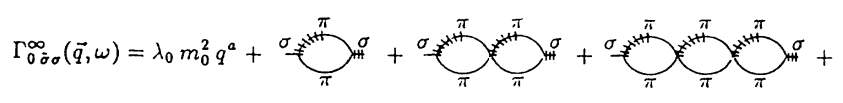

(b)

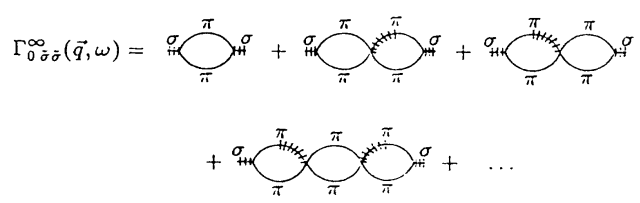

FIG. 2. Geometric series for the transverse two-point vertex functions in the coexistence limit.

point vertex functions is correctly reproduced by the spherical model limit $n \rightarrow \infty$. This renders our introductory heuristic arguments more precise. In fact, we can even deduce that the asymptotic fixed point must be of the form $u_{C}^{*} \propto 1 /(n-1)$, for the $n$-dependent factors in the series of Fig. 2 to cancel.

In the case of a conserved order parameter, i.e., model B with $a=2$, the previous results have to be taken with some caution, because the transformation (3.14b) cannot be performed for the $q=0$ component of the auxiliary fields. However, after inspection of (3.14a) into the dynamical functional, only one nonlinear term persists, namely, a $(\tilde{\pi} \pi \varphi)$ vertex, and because there is no $(\varphi \varphi)$ correlation propagator, no loop diagrams can be constructed. Hence there is no problem with the dynamical susceptibilities studied in this paper. Generally, one has to be careful with the limits $m_{0} \rightarrow \infty$ and $\mathbf{q} \rightarrow 0$, which do not commute. ${ }^{30}$

\section{RENORMALIZATION AND DISCUSSION OF THE FLOW EQUATIONS}

In this section we describe the general renormalization scheme and the specific modifications that have to be introduced for the description of the phase with spontaneously broken continuous symmetry. We explicitly calculate the renormalization constants to one-loop order and derive the renormalization-group equation. The flow equations are solved numerically and studied in the entire crossover region. We emphasize the scaling behavior and comment on the situation at the upper and lower critical dimensions.

\section{A. Dimensional regularization without $\epsilon$ expansion and generalized minimal subtraction}

Critical phenomena are characterized by typical infrared divergences on a subset of parameter space. In our case this critical surface is defined by $T \leq T_{c}, \widetilde{h}^{n}=0$, $\mathbf{q}=0$, and $\omega=0$. At $T_{c}$ itself we have the usual criticalpoint singularities, while below $T_{c}$ the coexistence anomalies appear. Hence we have to describe a crossover between situations of different critical behavior.
As the cutoffs in momentum space are pushed to infinity, for $d \geq 4$ dimensions also ultraviolet divergences arise. Within the dimensional regularization procedure by 't Hooft and Veltman, ${ }^{25}$ which has proved especially fruitful in the context of phase transitions, they show up as poles in $\epsilon=4-d$. The general idea of renormalization-group theory is now to exploit the fact that at $d_{c}=4$ these ultraviolet divergences are intimately connected with the physical infrared singularities. Generally, if $\tau_{0}$ is a variable characterizing the separation from the critical surface (note that here the $T_{c}$ shift is already included), the dimensionally regularized vertex functions may be written as

$$
\begin{aligned}
& \Gamma_{0}^{(N)}\left(u_{0}, \tau_{0}, d\right) \\
& \quad=\tau_{0}^{-d_{N} / 2}\left[1+\sum_{v=1}^{\infty} a_{v}^{(N)}(d)\left(\frac{u_{0} \tau_{0}^{-\epsilon / 2}}{\epsilon}\right)^{v}\right],
\end{aligned}
$$

which corresponds to a perturbation theory with the expansion parameter $u_{0} \tau_{0}^{-\epsilon / 2} / \epsilon .^{21}$ The $\epsilon$ poles sampling the ultraviolet divergences are now collected in the renormalization constants ( $Z$ factors), which define the connection between the bare fields or coupling constants (in$\operatorname{dex} 0$ ) and the renormalized quantities. From the $Z$ factors the anomalous dimensions are derived, which then describe the correct infrared behavior for $\tau_{0} \rightarrow 0$. The finite parts (for $\epsilon \rightarrow 0$ ), on the other hand, yield the amplitude functions. Via the renormalization-group equation, "naive" perturbation theory with small expansion parameters $\left(\tau_{0} \gg 1\right)$ can be mapped onto the critical theory displaying infrared singularities. By choosing an appropriate matching condition, the flow parameter is replaced by a physical scaling variable. After this procedure the correlation functions factorize into an exponent function characterized by an anomalous dimension and the amplitude function which displays scaling behavior when a fixed point of the renormalization group is approached. Following the arguments of Schloms and Dohm, ${ }^{21}$ for neither of these steps is an expansion with respect to $\epsilon$ necessary.

However, the perturbation series (4.1) usually diverges or is merely asymptotically convergent. To cope with this problem, one can either apply a suitable resummation analysis (as in Ref. 21) or use the $\epsilon$ expansion, which provides a sufficiently small expansion parameter. In our case now, we can take advantage of the fact that the perturbation expansion for the cumulants in the coexistence limit reduces to the zero- and one-loop contributions. Hence we do not have to use any further approximations and may refrain from $\epsilon$ expansion without any resummation procedure. In the vicinity of the critical point, of course, the one-loop results for the cumulants will only approximately reflect the physics.

A very elegant method to describe the crossover between critical and coexistence behavior is to modify the widely used minimal-subtraction prescription, following Amit and Goldschmidt ${ }^{18}$ and Lawrie. ${ }^{19,20}$ Let us examine, for example, the contribution to $G_{0 \widetilde{\sigma} \sigma}(\mathbf{q}, 0)$ constructed from a loop of two longitudinal modes (see Appendix B). Using dimensional regularization and Feyn- 
man parametrization, the integral for $q=\mu$ is proportional to

$$
\frac{A_{d} \mu^{-\epsilon}}{\epsilon} \int_{0}^{1}\left[\frac{1-x^{2}}{4}+\frac{m_{0}^{2}}{\mu^{2}}\right)^{-\epsilon / 2} d x .
$$

As long as $m_{0}$ is finite, there is an $\epsilon$ pole to be collected in the corresponding $Z$ factor. For $m_{0} \rightarrow \infty$, however, the integral vanishes and the ultraviolet singularity disappears. Now, if we write

$$
\begin{aligned}
\frac{A_{d} \mu^{-\epsilon}}{\epsilon} & \frac{1}{\left(1+m_{0}^{2} / \mu^{2}\right)^{\epsilon / 2}} \\
& \quad \times \int_{0}^{1}\left(\frac{1+m_{0}^{2} / \mu^{2}}{\left(1-x^{2}\right) / 4+m_{0}^{2} / \mu^{2}}\right)^{\epsilon / 2} d x,
\end{aligned}
$$

instead of $(4.2 \mathrm{a})$, obviously the parameter integral reduces to 1 in both limits $\epsilon \rightarrow 0$ and $m_{0} \rightarrow \infty$, and taking the prefactor as a contribution for the renormalization constant would correctly include the behavior in the coexistence limit. The $Z$ factors are then functions of the coupling constant $u_{0}$ and the mass parameter $m_{0} \cdot{ }^{18}$

In order to elucidate further how the specific infrared singularities caused by the Goldstone modes enter the theory, let us investigate the singular part of the typical integral $m_{0}^{2} \int_{k} 1 / k^{4}\left(m_{0}^{2}+k^{2}\right)$. As long as $m_{0}$ is finite, it is ultraviolet convergent and there is no $\epsilon$ pole. However, in the case $m_{0} \rightarrow \infty$, it reduces to the singular integral $\int_{k} 1 / k^{4}$. Indeed, using the generalized minimalsubtraction scheme, one gets

$$
\begin{array}{r}
\left.m_{0}^{2} \int_{k} \frac{1}{k^{4}\left(m_{0}^{2}+k^{2}\right)}\right|_{\text {sing }} \\
=\frac{A_{d} \mu^{-\epsilon}}{\epsilon}\left(1-\frac{1}{\left(1+m_{0}^{2} / \mu^{2}\right)^{\epsilon / 2}}\right),
\end{array}
$$

where the resulting pole for $\epsilon \rightarrow 0$ in the limit $m_{0} \rightarrow \infty$ can be eventually traced back to the infrared divergency of the integral.

Thus we can formulate our conditions for the generalized minimal-subtraction procedure: The renormalization constants have to be determined in such a way that the renormalized correlation functions are finite when $\epsilon \rightarrow 0$, both for finite $m_{0}$ and in the limit $m_{0} \rightarrow \infty$. We remark that a similar scheme has been successfully employed to investigate the crossover from the Heisenberg fixed point to asymptotic behavior dominated by the long-range dipolar interaction in both isotropic and uniaxial dipolar ferromagnets. ${ }^{31}$

\section{B. Renormalization constants for the isotropic models $A$ and $B$}

For finite $m_{0}$, simple dimensional analysis and power counting ${ }^{4}$ for the vertex function $\Gamma_{0 \tilde{N} N}\left(\left\{\mathbf{q}_{i}, \omega_{i}\right\}\right)$ with canonical dimension

$$
d_{\tilde{N} N}=-\frac{d}{2}(\tilde{N}+N-2)-\tilde{N}+N+2+a
$$

yields the primitive ultraviolet divergency

$$
\delta_{\tilde{N} N}=d_{\tilde{N} N}-(4-d) v_{4}-\frac{4-d}{2} v_{3}-a \tilde{N}
$$

for a diagram with $v_{4}$ four-vertices and $v_{3}$ three-vertices. Thus, for $d<2<4$, the contributions become less divergent with increasing orders of perturbation theory and also for higher $N$ when $\widetilde{N}=1$ is fixed. The theory is then called (super)renormalizable, and a finite number of $Z$ factors will be sufficient to cure the ultraviolet infinities.

To one-loop order, in fact, only $\Gamma_{020}, \Gamma_{011}, \Gamma_{012}$, and $\Gamma_{013}$ display singular behavior. With the aid of the Ward-Takahashi identities listed in Appendix A, we note that the different four-point functions are renormalized by the same $Z$ factor, and we shall not have to distinguish between several renormalized couplings $u$. Furthermore, the same is true for the three-point functions, and we realize that finite $\Gamma_{12}$ already imply finite $\Gamma_{13}$. Finally, we see that it is even sufficient to eliminate the singularities within the two-point functions. Of course, this is true only in the ordered phase where $\Gamma_{\tilde{\pi} \pi} \neq \Gamma_{\tilde{\sigma} \sigma}$, but here it provides a considerable facilitation.

In the limit $m_{0} \rightarrow \infty$, these arguments fail because the analysis of primitive divergencies is not sufficient any more (longitudinal propagators $1 / m_{0}^{2}$ may compensate factors $m_{0}$ in the three-vertices). But as we have seen in the last section, the asymptotic theory can be treated exactly, and only the transverse loops persist in the coexistence limit. Their infinities can obviously be absorbed into renormalized quantities $m$ and $u$, with $u / u_{0}$ $\propto m^{2} / m_{0}^{2}$ : From (3.17) and (3.18) this is clear for the two-point functions, and higher correlations within the Gaussian theory always factorize in products of these.

We now define the renormalized fields according to

$$
\begin{aligned}
& \tilde{\pi}^{\alpha}=Z_{\tilde{\pi}}^{1 / 2} \widetilde{\pi}_{0}^{\alpha}, \\
& \widetilde{\sigma}=Z_{\widetilde{\sigma}}^{1 / 2} \widetilde{\sigma}_{0}, \\
& \pi^{\alpha}=Z_{\pi}^{1 / 2} \pi_{0}^{\alpha}, \\
& \sigma=Z_{\sigma}^{1 / 2} \sigma_{0},
\end{aligned}
$$

taking careful account of the symmetry breaking. Similarly, one has to distinguish between the transverse and longitudinal timescales $\lambda_{1}$ and $\lambda_{\|}$, being attached to a $\widetilde{\pi}^{\alpha}$ or $\widetilde{\sigma}$ field, respectively. The dimensionless renormalized parameters thus read

$$
\begin{aligned}
& \lambda_{\perp}=Z_{\lambda_{\perp}}^{-1} \lambda_{0}, \\
& \lambda_{\|}=Z_{\lambda_{\|}^{-1}}^{-1} \lambda_{0}, \\
& m^{2}=Z_{m}^{-1} m_{0}^{2} \mu^{-2}, \\
& u=Z_{u}^{-1} u_{0} A_{d} \mu^{-\epsilon} .
\end{aligned}
$$

The program according to our general regularization procedure and in view of the coexistence limit is then to ensure that the renormalized cumulants

$$
G_{\tilde{\pi} \pi}^{c}(\mathbf{q}, \omega)=\left(Z_{\tilde{\pi}} Z_{\pi}\right)^{1 / 2} G_{0 \pi \pi}^{c}(\mathbf{q}, \omega)
$$




$$
\begin{aligned}
& G_{\tilde{\sigma} \sigma}^{c}(\mathbf{q}, \omega)=\left(Z_{\tilde{\sigma}} Z_{\sigma}\right)^{1 / 2} G_{0 \widetilde{\sigma} \sigma}^{c}(\mathbf{q}, \omega) \\
& G_{\pi \pi}^{c}(\mathbf{q}, \omega)=Z_{\pi} G_{0 \pi \pi}^{c}(\mathbf{q}, \omega) \\
& G_{\sigma \sigma}^{c}(\mathbf{q}, \omega)=Z_{\sigma} G_{0 \sigma \sigma}^{c}(\mathbf{q}, \omega)
\end{aligned}
$$

are convergent for $\epsilon \rightarrow 0$ both for finite $m$ and in the limit $m \rightarrow \infty$, i.e., along the entire coexistence curve.

The renormalization constants introduced in Eqs. (4.5) and (4.6) are not completely independent. From the renormalized analog to (3.2) for the spontaneous order parameter, it follows that

$$
Z_{u}=Z_{m} Z_{\sigma}
$$

Two other important relations stem from the renormalized version of the fluctuation-dissipation theorem (2.21b), namely, ${ }^{4}$

$$
\begin{aligned}
& Z_{\lambda_{\perp}}=Z_{\widetilde{\pi}}^{1 / 2} Z_{\pi}^{-1 / 2}, \\
& Z_{\lambda_{\|}}=Z_{\tilde{\sigma}}^{1 / 2} Z_{\sigma}^{-1 / 2} .
\end{aligned}
$$

Thus we have to determine five independent $Z$ factors, e.g., by rendering the quantities $\partial_{\omega} \Gamma_{\tilde{\pi} \pi}(\mathbf{q}, \omega)$, $\partial_{q^{2}}\left(\frac{1}{2} \partial_{q^{2}}\right)^{a / 2} \Gamma_{\tilde{\pi} \pi}(\mathbf{q}, \omega), \quad \partial_{\omega} \Gamma_{\widetilde{\sigma} \sigma}(\mathbf{q}, \omega), \quad\left(\partial_{q^{2}}\right)^{a / 2} \Gamma_{\widetilde{\sigma} \sigma}(\mathbf{q}, \omega)$, $\partial_{q^{2}}\left(\frac{1}{2} \partial_{q^{2}}\right)^{a / 2} \Gamma_{\tilde{\sigma} \sigma}(\mathbf{q}, \omega), \partial_{q^{2}}^{a / 2} \Gamma_{\tilde{\pi} \tilde{\pi}}(\mathbf{q}, \omega)$, and $\partial_{q^{2}}^{a / 2} \Gamma_{\tilde{\sigma} \tilde{\sigma}}(\mathbf{q}, \omega)$ finite at the normalization point NP: $q=\mu, \omega=0$ (the redundant two conditions may serve as a check). Because of the close connection with the response functions according to $(2.19 \mathrm{~b})$, it is clear that the resulting $Z$ factors belonging to the static quantities will be identical to those already derived within the static theory. ${ }^{19}$

We finally remark that in the case of model B with conserved order parameter the number of independent $Z$ factors is further reduced, because the momentum dependence of the relaxational vertex $\propto q^{2}$ leads to the disappearance of the loop contributions for $\Gamma_{0 \tilde{\pi} \pi}(0, \omega)$ and $\Gamma_{0 \tilde{\sigma} \sigma}(0, \omega)$, from which we conclude ${ }^{4}$

$$
\begin{aligned}
a=2: & \left(Z_{\tilde{\pi}} Z_{\pi}\right)^{1 / 2}=1, \\
& \left(Z_{\widetilde{\sigma}} Z_{\sigma}\right)^{1 / 2}=1 .
\end{aligned}
$$

Using only the one-loop diagrams (see Appendix B), we expect the field renormalizations to vanish, and indeed one finds, both for model A and B,

$$
Z_{\tilde{\pi}}=Z_{\widetilde{\sigma}}=Z_{\pi}=Z_{\sigma}=1,
$$

implying

$$
Z_{\lambda_{1}}=Z_{\lambda_{\|}}=1
$$

so that the only nontrivial $Z$ factor is the one for the mass parameter and coupling constant:

$$
\begin{aligned}
Z_{m}\left(u_{0}, m_{0}\right)= & Z_{u}\left(u_{0}, m_{0}\right) \\
= & 1+\frac{n-1}{6 \epsilon} u_{0} A_{d} \mu^{-\epsilon} \\
& +\frac{3}{2 \epsilon} \frac{u_{0} A_{d} \mu^{-\epsilon}}{\left(1+m_{0}^{2} / \mu^{2}\right)^{\epsilon / 2}} .
\end{aligned}
$$

For $m_{0}=0$ this is exactly the familiar renormalization constant in the critical region, while in the coexistence limit $m_{0} \rightarrow \infty$ the weight of the effective critical fluctuations is reduced from $n+8$ to $n-1$, the number of Goldstone modes. We emphasize again that asymptotically the results (4.11) become exact, while in the crossover region they are correct up to terms of the order $u_{0}^{2} /\left(1+m_{0}^{2} / \mu^{2}\right)^{\epsilon / 2}$.

\section{Renormalization-group equation and discussion of the flow equations}

The renormalization-group equations connect the uncritical theory which can be treated perturbationally with the critical theory displaying infrared divergences. For their derivation we remark that the unrenormalized $N$ point functions do not depend on the scale $\mu$ defined by the renormalization point $N P$ :

$$
\left.\mu \frac{d}{d \mu}\right|_{0} G_{0 \tilde{N} N}^{c}=0 .
$$

This leads to the following partial differential equations for the two-point cumulants:

$$
\begin{aligned}
& {\left[\mu \frac{\partial}{\partial \mu}+\zeta_{\lambda_{\perp}} \lambda_{\perp} \frac{\partial}{\partial \lambda_{\perp}}+\zeta_{\lambda_{\|}} \lambda_{\|} \frac{\partial}{\partial \lambda_{\|}}+\zeta_{m} m^{2} \frac{\partial}{\partial m^{2}}\right.} \\
& \left.+\beta_{u} \frac{\partial}{\partial u}-\frac{1}{2} \zeta_{\tilde{\pi}}-\frac{1}{2} \zeta_{\pi}\right] G_{\tilde{\pi} \pi}^{c}=0, \\
& {\left[\mu \frac{\partial}{\partial \mu}+\zeta_{\lambda_{\perp}} \lambda_{\perp} \frac{\partial}{\partial \lambda_{\perp}}+\zeta_{\lambda_{\|}} \lambda_{\|} \frac{\partial}{\partial \lambda_{\|}}+\zeta_{m} m^{2} \frac{\partial}{\partial m^{2}}\right.} \\
& \left.+\beta_{u} \frac{\partial}{\partial u}-\frac{1}{2} \zeta_{\tilde{\sigma}}-\frac{1}{2} \zeta_{\sigma}\right] G_{\tilde{\sigma} \sigma}^{c}=0, \\
& {\left[\mu \frac{\partial}{\partial \mu}+\zeta_{\lambda_{\perp}} \lambda_{\perp} \frac{\partial}{\partial \lambda_{\perp}}+\zeta_{\lambda_{\|}} \lambda_{\|} \frac{\partial}{\partial \lambda_{\|}}+\zeta_{m} m^{2} \frac{\partial}{\partial m^{2}}\right.} \\
& \left.+\beta_{u} \frac{\partial}{\partial u}-\zeta_{\pi}\right] G_{\pi \pi}^{c}=0, \\
& {\left[\mu \frac{\partial}{\partial \mu}+\zeta_{\lambda_{\perp}} \lambda_{\perp} \frac{\partial}{\partial \lambda_{\perp}}+\zeta_{\lambda_{\|}} \lambda_{\|} \frac{\partial}{\partial \lambda_{\|}}+\zeta_{m} m^{2} \frac{\partial}{\partial m^{2}}\right.} \\
& \left.+\beta_{u} \frac{\partial}{\partial u}-\zeta_{\sigma}\right] G_{\sigma \sigma}^{c}=0,
\end{aligned}
$$

where Wilson's $\zeta$ and $\beta$ functions

$$
\begin{aligned}
& \zeta_{\tilde{\pi}}=\left.\mu \frac{\partial}{\partial \mu}\right|_{0} \ln Z_{\tilde{\pi}}, \\
& \zeta_{\tilde{\sigma}}=\left.\mu \frac{\partial}{\partial \mu}\right|_{0} \ln Z_{\tilde{\sigma}}, \\
& \zeta_{\pi}=\left.\mu \frac{\partial}{\partial \mu}\right|_{0} ^{\ln Z_{\pi}},
\end{aligned}
$$




$$
\begin{aligned}
& \zeta_{\sigma}=\left.\mu \frac{\partial}{\partial \mu}\right|_{0} \ln Z_{\sigma}, \\
& \zeta_{\lambda_{\perp}}=\left.\mu \frac{\partial}{\partial \mu}\right|_{0} \ln \frac{\lambda_{\perp}}{\lambda_{0}}, \\
& \zeta_{\lambda_{\|}}=\left.\mu \frac{\partial}{\partial \mu}\right|_{0} \ln \frac{\lambda_{\|}}{\lambda_{0}}, \\
& \zeta_{m}=\left.\mu \frac{\partial}{\partial \mu}\right|_{0} \ln \frac{m^{2}}{m_{0}^{2}}, \\
& \beta_{u}=\left.\mu \frac{\partial}{\partial \mu}\right|_{0} ^{u}
\end{aligned}
$$

have been introduced (see, e.g., Ref. 24).

The partial differential equations (4.13) are readily solved with the method of characteristics. Defining the characteristics as

$$
\mu(l)=\mu l \text {, }
$$

the flow-dependent couplings are given by the set of firstorder ordinary differential equations

$$
\begin{aligned}
& l \frac{d \lambda_{\perp}(l)}{d l}=\lambda_{\perp}(l) \zeta_{\lambda_{1}}(l), \\
& l \frac{d \lambda_{\|}(l)}{d l}=\lambda_{\|}(l) \zeta_{\lambda_{\|}}(l), \\
& l \frac{d m(l)}{d l}=\frac{1}{2} m(l) \zeta_{m}(l), \\
& l \frac{d u(l)}{d l}=\beta_{u}(l),
\end{aligned}
$$

with the starting conditions $\lambda_{\perp}(1)=\lambda_{\perp}, \lambda_{\|}(1)=\lambda_{\|}$, $m(1)=m$, and $u(1)=u$. The solutions of (4.13) then read

$G_{\tilde{\pi} \pi}^{c}\left(\mu, \lambda_{\perp}, \lambda_{\|}, m, u,\{\mathbf{q}\},\{\omega\}\right)=\exp \left[-\frac{1}{2} \int_{1}^{l}\left[\zeta_{\tilde{\pi}}\left(l^{\prime}\right)+\zeta_{\pi}\left(l^{\prime}\right)\right] \frac{d l^{\prime}}{l^{\prime}}\right] G_{\tilde{\pi} \pi}^{c}\left(\mu l, \lambda_{\perp}(l), \lambda_{\|}(l), m(l), u(l),\{\mathbf{q} / \mu l\},\left\{\omega /(\mu l)^{2}\right\}\right)$,

$G_{\tilde{\sigma} \sigma}^{c}\left(\mu, \lambda_{1}, \lambda_{\|}, m, u,\{\mathbf{q}\},\{\omega\}\right)=\exp \left[-\frac{1}{2} \int_{1}^{l}\left[\zeta_{\tilde{\sigma}}\left(l^{\prime}\right)+\zeta_{\sigma}\left(l^{\prime}\right)\right] \frac{d l^{\prime}}{l^{\prime}}\right] G_{\tilde{\sigma} \sigma}^{c}\left(\mu l, \lambda_{1}(l), \lambda_{\|}(l), m(l), u(l),\{\mathbf{q} / \mu l\},\left\{\omega /(\mu l)^{2}\right\}\right)$,

$G_{\pi \pi}^{c}\left(\mu, \lambda_{\perp}, \lambda_{\|}, m, u,\{\mathbf{q}\},\{\omega\}\right)=\exp \left[-\int_{1}^{l} \zeta_{\pi}\left(l^{\prime}\right) \frac{d l^{\prime}}{l^{\prime}}\right] G_{\pi \pi}^{c}\left(\mu l, \lambda_{\perp}(l), \lambda_{\|}(l), m(l), u(l),\{\mathbf{q} / \mu l\},\left\{\omega /(\mu l)^{2}\right\}\right)$

$G_{\sigma \sigma}^{c}\left(\mu, \lambda_{\perp}, \lambda_{\|}, m, u,\{\mathbf{q}\},\{\omega\}\right)=\exp \left[-\int_{1}^{l} \zeta_{\sigma}\left(l^{\prime}\right) \frac{d l^{\prime}}{l^{\prime}}\right] G_{\sigma \sigma}^{c}\left(\mu l, \lambda_{\perp}(l), \lambda_{\|}(l), m(l), u(l),\{\mathbf{q} / \mu l\},\left\{\omega /(\mu l)^{2}\right\}\right)$

We shall evaluate the scaling functions from (4.18) in the following section with a suitable choice of a matching condition relating the flow parameter $l$ with a physical scaling variable, ensuring that (i) one of the variables on the RHS of (4.18) is fixed to a sufficiently large value in order that perturbation theory is applicable, and (ii) $l \rightarrow 0$ describes the asymptotic limit.

But let us first study the flow equations (4.17). The asymptotic behavior sets in when a fixed point of the renormalization group is approached. The fixed points are given by the zeros of the $\beta$ function (4.15d),

$$
\beta_{u}\left(u^{*}, m\right)=0,
$$

for then $u=u^{*}=$ const. They are called (infrared) stable if

$$
\left.\frac{\partial \beta_{u}(u, m)}{\partial u}\right|_{u=u^{*}}>0
$$

is satisfied, because in this case from any starting value $u(1)$ in the vicinity of $u^{*}$ the flow runs into the fixed point when $\quad l \rightarrow 0$. The corresponding values of $\zeta_{\lambda}^{*}$ $=\zeta_{\lambda_{1}}\left(u^{*}, m\right), \zeta_{\lambda_{\|}}^{*}=\zeta_{\lambda_{\|}}\left(u^{*}, m\right)$, and $\zeta_{m}^{*}=\zeta_{m}\left(u^{*}, m\right)$ define the anomalous dimensions of the time scales and the mass parameter.

From the $Z$ factors (4.11), we calculate the Wilson functions to one-loop order for the relaxational models to be

$\zeta_{\tilde{\pi}}=\zeta_{\tilde{\sigma}}=\zeta_{\pi}=\zeta_{\sigma}=0$

$\zeta_{\lambda_{\perp}}=\zeta_{\lambda_{\|}}=0$

$\zeta_{m}(u, m)=-2+\frac{n-1}{6} u+\frac{3}{2} \frac{u}{\left(1+m^{2}\right)^{1+\epsilon / 2}}$,

$\beta_{u}(u, m)=u\left[-\epsilon+\frac{n-1}{6} u+\frac{3}{2} \frac{u}{\left(1+m^{2}\right)^{1+\epsilon / 2}}\right]$

In the coexistence limit $m \rightarrow \infty$, these results become ex- 
act, while at the critical point and in the crossover region they are correct up to terms of the order $u^{2} /\left(1+m^{2}\right)^{1+\epsilon / 2}$.

At $T_{c}(m=0)$ we find the unstable Gaussian fixed point $u_{G}^{*}=0$ with the canonical mass dimension $\zeta_{m G}^{*}=-2$ and the stable nontrivial Heisenberg fixed point

$$
\begin{aligned}
& u_{H}^{*}=\frac{6 \epsilon}{n+8}, \\
& \zeta_{m H}^{*}=-2+\epsilon
\end{aligned}
$$

(in one-loop approximation), governing the critical regime. On the contrary, for $m \rightarrow \infty$, we still have the Gaussian fixed point and, additionally, the stable coexistence fixed point

$$
\begin{aligned}
& u_{C}^{*}=\frac{6 \epsilon}{n-1}, \\
& \zeta_{m C}^{*}=-2+\epsilon,
\end{aligned}
$$

which was found by Lawrie. ${ }^{19}$ Note that both the Heisenberg and coexistence fixed points are characterized by the same anomalous dimension $\zeta_{m}^{*}$; this is a consequence of the general one-loop result $Z_{m}=Z_{u}$ and is valid for any nontrivial fixed point. For $0 \leq \epsilon<2$ the renormalized mass parameter does indeed flow to infinity, $m(l)^{2} \propto l^{-2+\epsilon}$. Hence the asymptotic limit is really described by a diverging longitudinal mass, and the discussion of Sec. III C applies.

Further information about the crossover from $u_{H}^{*}$ to $u_{C}^{*}$ can be obtained by solving the coupled flow equations $(4.17 \mathrm{c})$ and $(4.17 \mathrm{~d})$ with $(4.21 \mathrm{c})$ and $(4.21 \mathrm{~d})$ numerically. In Fig. 3 we depict the flow of $u(l)$ in the case $n=2$ and $\epsilon=1$ for several starting values in the range $0.2 \leq u(1) \leq 1.2$ and $m(1)=0.01,0.1,1.0$, respectively. For small masses $m(1)<0.1$, the flow does not directly run into the asymptotic behavior $u_{C}^{*}=6$, but approaches a quasiuniversal plateau first, which corresponds to the usual critical theory in the vicinity of $T_{c}$ characterized by $u_{H}^{*}=0.6$. Of course, eventually, the coexistence limit is reached. Only for large $m(1)>0.1$ has the plateau more or less disappeared. The onset of the crossover is shifted to smaller values of $l$ if $m(1)$ is decreased.

In Fig. 4 we display two flow diagrams (for $n=2$ and 3 , respectively, with $\epsilon=1)$ showing $u(l)$ vs $m(l)$ or, rather, $m(l)^{2} /\left[1+m(l)^{2}\right]$, which assumes values in the interval $[0 ; 1]$. The Heisenberg fixed point is clearly attractive for the parameter flow if $m(1)$ is small, even for large $u(1)$. For very small $u(1)$ even the influence of the Gaussian fixed point is noticeable. The curve connecting $u_{H}^{*}$ and $u_{C}^{*}$ defines a separatrix in the flow diagram; it describes the universal crossover from critical to the coexistence behavior dominated by the Goldstone modes.

It is possible to recover this universal crossover in the flow of the coupling parameter $u(l)$ for small starting values of $m$. Because of (4.8) and with the one-loop result $Z_{\sigma}=1$, a renormalization-group invariant can be identified, namely,

$$
\frac{m(l)^{2}}{u(l)} l^{2-\epsilon}=\frac{m(1)^{2}}{u(1)} ;
$$

hence, wherever $u(l)$ is approximately constant, we have
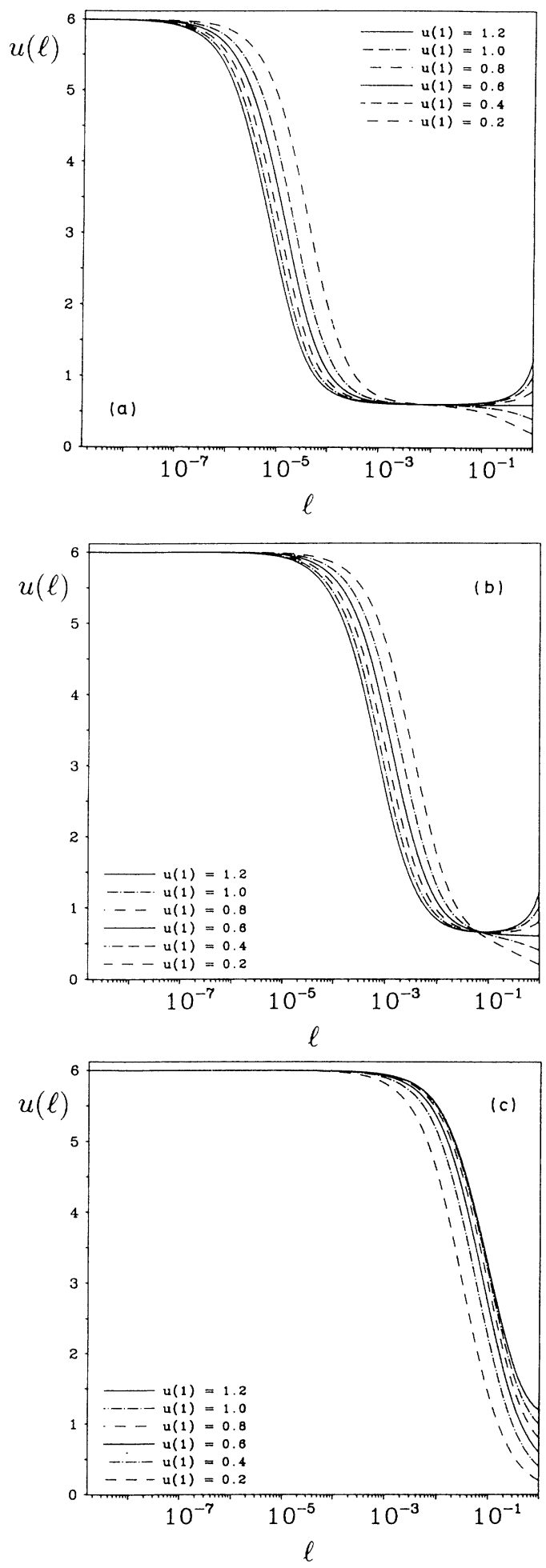

FIG. 3. Flow of the coupling $u(l)$ for several starting values $0.2 \leq u(1) \leq 1.2$ and $m(1)=$ (a) 0.01 , (b) 0.1 , and (c) $1.0 ; n=2$, $\epsilon=1$. 

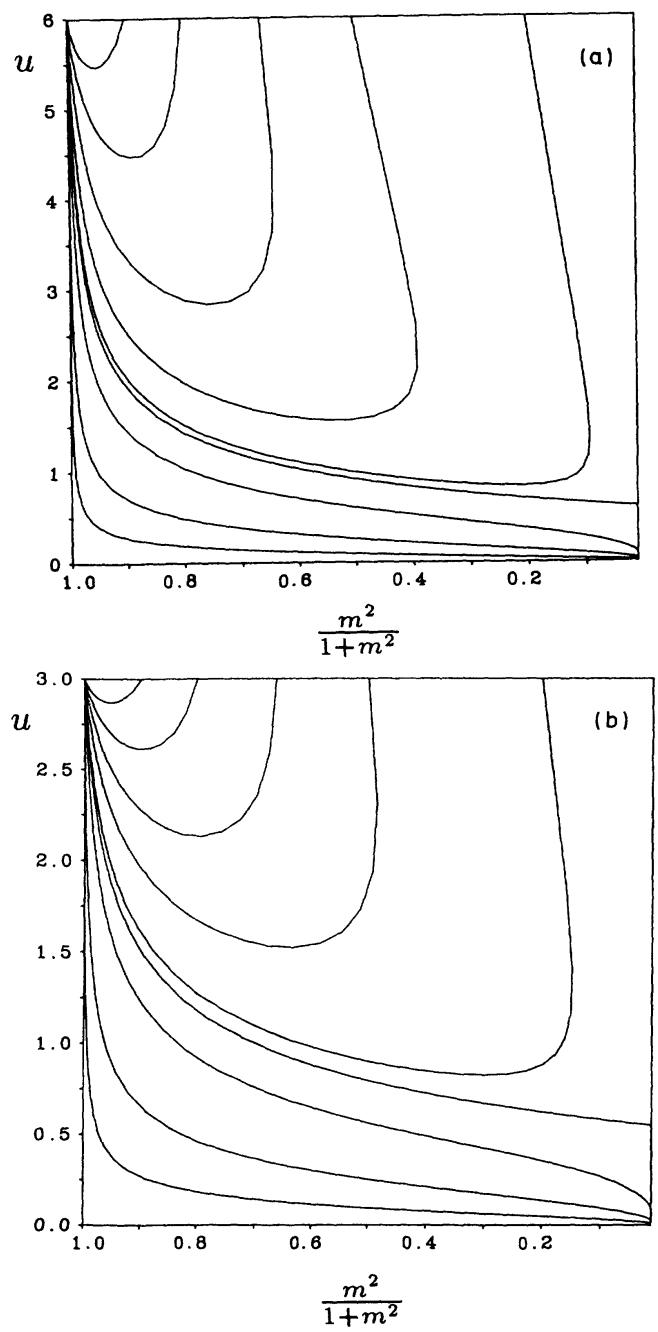

FIG. 4. Flow diagrams $u(l)$ vs $m(l)^{2} /\left[1+m(l)^{2}\right]$ for $n=$ (a) 2 and (b) $3 ; \epsilon=1$.

$$
m(l)^{2} \propto\left[\frac{l}{m(1)^{2 /(2-\epsilon)}}\right]^{-2+\epsilon} .
$$

Remarkably, the numerical analysis shows that for

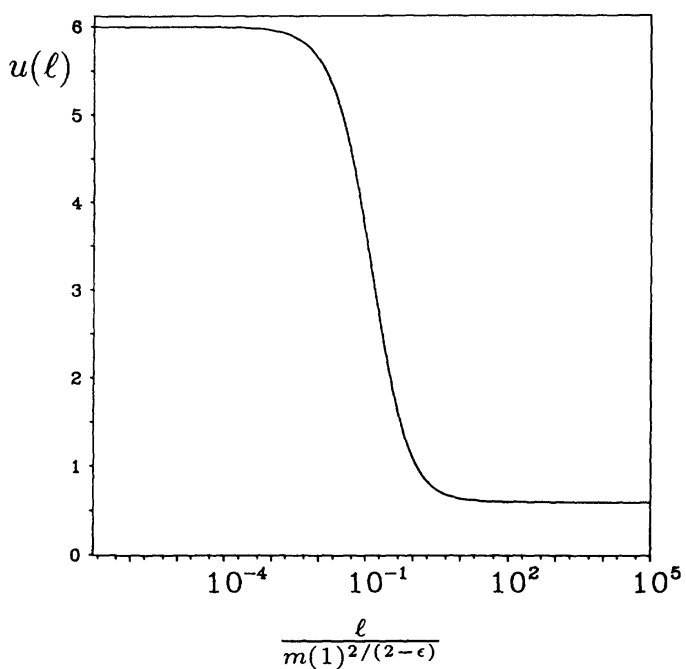

FIG. 5. Universal crossover: $u(l)$ vs the scaling variable $l / m(1)^{2 /(2-\epsilon)}$ for $m(1)<0.1 ; n=2$ and $\epsilon=1$ as in Fig. 3.

$m(1)<1$ the flows even coincide in the crossover region, if $u(l)$ is plotted against

$$
x=\frac{l}{m(1)^{2 /(2-\epsilon)}}
$$

[with fixed $u(1)$ ], which we therefore identify as the relevant scaling variable (Fig. 5).

We end this section with an approximate solution of the flow equations (4.21c) and (4.21d) and some comments on what happens at the upper and lower critical dimensions. The previous discussion was valid in the range $2<d<4$. To get a qualitatively correct picture, we could have inserted (4.24b) for the flow of the mass parameter into the differential equation (4.17d), which then is of the Bernoulli type and is readily solved with the substitution $w(l)=1 / u(l)$. Up to a quadrature the result is

$$
u(l)=\frac{u l^{-\epsilon}}{1-u \int_{1}^{l}\left[(n-1) / 6+\frac{3}{2}\left(1+m^{2} l^{\prime-2+\epsilon}\right)^{-1-\epsilon / 2}\right] l^{\prime-1-\epsilon} d l^{\prime}}
$$

with $u=u$ (1) and $m=m$ (1). Substituting (4.25), we find

$$
u(x)=\frac{u}{m^{2 \epsilon /(2-\epsilon)} x^{\epsilon}-u x^{\epsilon} \int_{1 / m^{2 /(2-\epsilon)}}^{x}\left[(n-1) / 6+\frac{3}{2}\left(1+x^{\prime-2+\epsilon}\right)^{-1-\epsilon / 2}\right] x^{\prime-1-\epsilon} d x^{\prime}},
$$

from which the scaling behavior with respect to the variable $x$ is obvious for small values of $m$ when the first term in the denominator is negligible.

In the case $\epsilon=1,(4.26 \mathrm{a})$ becomes

$$
u(l)=\frac{u l^{-1}}{1-(n-1) / 6 u(1-1 / l)-\left(3 u / m^{2}\right)\left\{\left[l /\left(m^{2}+l\right)\right]^{1 / 2}-1 /\left(m^{2}+1\right)^{1 / 2}\right\}},
$$


which yields a similar behavior as displayed in Figs. 3-5; i.e., in the limit $l \rightarrow 0$, the coexistence fixed point $u_{c}^{*}=6 /(n-1)$ is approached, while the crossover point is mainly determined by the initial value $m$.

At the upper critical dimension $d_{c}=4(\epsilon=0)$, on the other hand, we find that $u(l) \rightarrow 0$ according to

$$
u(l)=\frac{u}{1-(n-1) / 6 u \ln l-\frac{3}{4} u \ln \left[\left(m^{2}+l^{2}\right) /\left(m^{2}+1\right)\right]} .
$$

This is exactly the expected Gaussian theory with logarithmic corrections.

In two dimensions $(\epsilon=2)$, however, the situation becomes quite different. The solution (4.26a) now reads

$$
u(l)=\frac{u}{1+\left[(n-1) / 12+\frac{3}{4} 1 /\left(1+m^{2}\right)^{2}\right] u\left(1-l^{2}\right)}
$$

i.e., $u(l)$ approaches a finite nonuniversal constant value. The reason is that according to $(4.24 \mathrm{~b})$ the longitudinal mass $m(l)$ no longer diverges and $u(l) \propto m(l)^{2}[\mathrm{Eq}$. (4.24a)]. In fact, for low values of $m$, the isotropic Heisenberg fixed point (4.22) is approached [see Eq. (4.26b)]. This merely indicates that the assumption of spontaneous symmetry breaking in connection with a uniform order parameter leads to inconsistencies for $d=2$. These conclusions are in accord with the Mermin-Wagner theorem that forbids long-range order in two dimensions if there is a global continuous symmetry and only short-range interaction. ${ }^{27,28}$

\section{DYNAMICAL SUSCEPTIBILITIES AND CORRELATION FUNCTIONS}

We are now able to calculate the dynamical response functions for the entire crossover region within the renormalized theory. We start with the static susceptibilities and then proceed to the dynamical correlation functions for both model A, corresponding to purely dissipative relaxation, and model B, with conserved order parameter and hence characterized by diffusive behavior.

\section{A. Static susceptibilities}

In order to determine the static and dynamical scaling functions, we evaluate the one-loop diagrams for the two-point cumulants, which can be easily derived from the one-loop vertex functions listed in Appendix B. The results of the unrenormalized perturbation expansion now have to be mapped onto the critical theory by introducing renormalized quantities and using the renormalization-group equations (4.18). We then find that because of the transverse loops, characteristic infrared singularities may persist. However, by choosing a suitable matching condition, one of the arguments (or a combination of several variables) on the RHS of Eqs. (4.18) can be fixed at a sufficiently large value such that the usual perturbation theory is applicable. In order to ensure that $l \rightarrow 0$ describes the asymptotic behavior, which in our case represents the coexistence limit, we identify the flow parameter $l^{2}$ with the absolute value of the inverse transverse susceptibility to zero-loop order,

$$
l^{2}=\left|\frac{q^{2}}{\mu^{2}}-\frac{i \omega}{\lambda \mu^{2}(q / \mu)^{a}}\right|,
$$

which measures the distance from the coexistence critical surface. Thus the infrared singularities are effectively exponentiated, while the remaining scaling function is finite for vanishing momenta and frequencies.

In the static limit $\omega=0$, the general matching condition (5.1) eliminating the infrared singularity $\propto q^{-\epsilon}$ caused by the transverse loop reduces to

$$
l=\frac{q}{\mu} .
$$

Using (5.2) and the fact that for low values of $m(1)$, i.e., close to the transition temperature, the scaling variable introduced in the preceding section should be proportional to the product of wave number $q$ and correlation length $\xi$,

$$
\frac{l}{m(1)^{2 /(2-\epsilon)}} \propto \frac{q}{\bar{\phi}^{2 /(2-\epsilon)}} \propto q \xi,
$$

together with the usual definition of the critical exponents $v$ and $\beta$ according to $\xi \propto|\tau|^{-v}$ and $\bar{\phi} \propto|\tau|^{\beta}$, respectively, we find the scaling relation

$$
v=\frac{2 \beta}{2-\epsilon} \text {. }
$$

This agrees with the general result $v=2 \beta /(2-\epsilon+\eta),{ }^{25}$ because $\eta=0$ in one-loop approximation. The scaling behavior with respect to the variable $(4.25)$ is therefore intimately connected with the validity of $(5.3 \mathrm{~b})$ in the vicinity of $T_{c}$. For example, using the $\epsilon$ expansion, one finds $\beta=\frac{1}{2}-3 \epsilon / 2(n+8)+O\left(\epsilon^{2}\right),{ }^{25}$ and from (5.3b) we correctly obtain

$$
v=\frac{1}{2}+\frac{(n+2) \epsilon}{4(n+8)}+O\left(\epsilon^{2}\right)
$$

Similarly, for $n \rightarrow \infty$, we have exactly $\beta=\frac{1}{2}$, and hence $v=1 /(2-\epsilon)$, which is precisely the result for the spherical model. ${ }^{7}$

Using

$$
Z_{m}(l)=1+\frac{n-1}{6} u(l)+\frac{3}{2} \frac{u(l)}{\left[1+m(l)^{2}\right]^{\epsilon / 2}},
$$

we arrive at the following expressions for the transverse and longitudinal static susceptibilities, respectively, in three dimensions $(\epsilon=1)$ : 


$$
\begin{aligned}
& \chi_{T}^{-1}(\mathbf{q}, 0)=\left.\frac{\mu^{2} l^{2}}{1-\frac{1}{6} u(l) m(l)\left[2-m(l)\left[\frac{\pi}{2}+\arcsin \frac{1-m(l)^{2}}{1+m(l)^{2}}\right]\right)}\right|_{l=q / \mu}, \\
& \chi_{L}^{-1}(\mathbf{q}, 0)=\left.\frac{\mu^{2} l^{2}\left[1+m(l)^{2} Z_{m}(l)\right]}{1+\frac{u(l) m(l)^{2}}{1+m(l)^{2}}\left[\frac{n-1}{12} \pi+\frac{3}{2} \arcsin \frac{1}{\left[1+4 m(l)^{2}\right]^{1 / 2}}\right]}\right|_{l=q / \mu},
\end{aligned}
$$

valid both for $a=0$ and $a=2$, of course. These results have the general scaling form

$$
\chi_{T / L}^{-1}(\tau, \mathbf{q}, 0)=q^{2-\eta} \hat{\chi}_{T / L}^{-1}(q \xi),
$$

with $\eta=0$, and the universal functions $\hat{\chi}_{T / L}(q \xi)$ describe the crossover from critical to coexistence behavior. Figure 6 shows a double-logarithmic plot of the inverse static response functions for $n=2$, comparing the results as derived from the cumulants $(C)$ [Eq. (5.5)] with those from the vertex functions $(V)$ [to be obtained from (5.5)
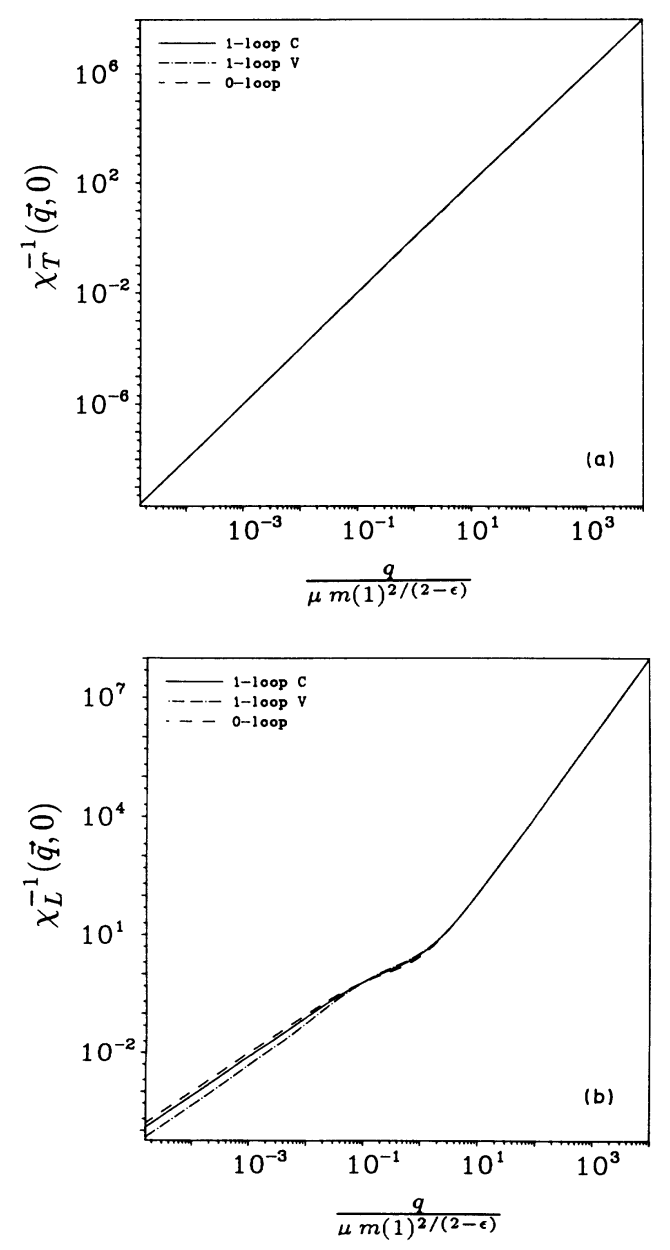

FIG. 6. Inverse (a) transverse and (b) longitudinal static susceptibilities for $\epsilon=1$ and $n=2$. by expansion to first order in $u$ ] and the zero-loop expressions $(u=0)$. In either case, however, the numerical solutions of the one-loop flow equations (4.17c) and (4.17d) with (4.21c) and (4.21d) have been inserted, and a small initial value $m(1)<0.1$ has been assumed in order to study the universal crossover. While always

$$
\chi_{T}^{-1}(\mathbf{q}, 0) \propto q^{2},
$$

there is a drastic change in the leading behavior of the longitudinal susceptibility from being proportional to $q^{2}$ to

$$
\chi_{L}^{-1}(\mathbf{q}, 0) \propto q^{\epsilon}
$$

across a region with even lower slope at around $q / \mu m(1)^{2 /(2-\epsilon)} \approx 1$. This is a first example of a coexistence anomaly, for in the absence of the original $\mathrm{O}(n)$ symmetry the longitudinal susceptibility would simply attain a finite value, while it diverges because of the effect of the Goldstone modes. We remark that formally this singularity is to be traced back to the anomalous dimension of the longitudinal mass [Eq. (4.23b)] for $m(l)^{2} l^{2} \propto l^{\epsilon}$. Note that the leading asymptotic behavior is factored out of the scaling function and is no more contained in any of the loop diagrams. Therefore the renormalized zero-loop results already describe the situation qualitatively very well, if the flow-dependent parameters resulting from the full one-loop flow equations $(4.17 \mathrm{c})$, (4.17d), (4.21c), and (4.21d) are used.

The $q^{-\epsilon}$ behavior was discussed within a renormalization-group approach by Mazenko, ${ }^{14}$ using arguments of the $1 / n$ expansion. Another exponentiation of the Goldstone singularities was achieved by Schäfer and Horner through a more sophisticated partial summation of the perturbation series. ${ }^{15}$ The Goldstone anomalies then were fully embedded into the concept of renormalization-group theory for crossover phenomena by Lawrie. ${ }^{19}$ However, he primarily examined the coexistence limit, using only an approximate solution of the flow equations in the $\epsilon$ expansion. Our scheme thus goes beyond Lawrie's approach in investigating the crossover region more accurately.

This is most conveniently done by introducing the quantities

$$
2-\eta_{\mathrm{eff}}^{T / L}=\frac{\partial \ln \chi_{T / L}^{-1}(\mathbf{q}, 0)}{\partial \ln q}
$$



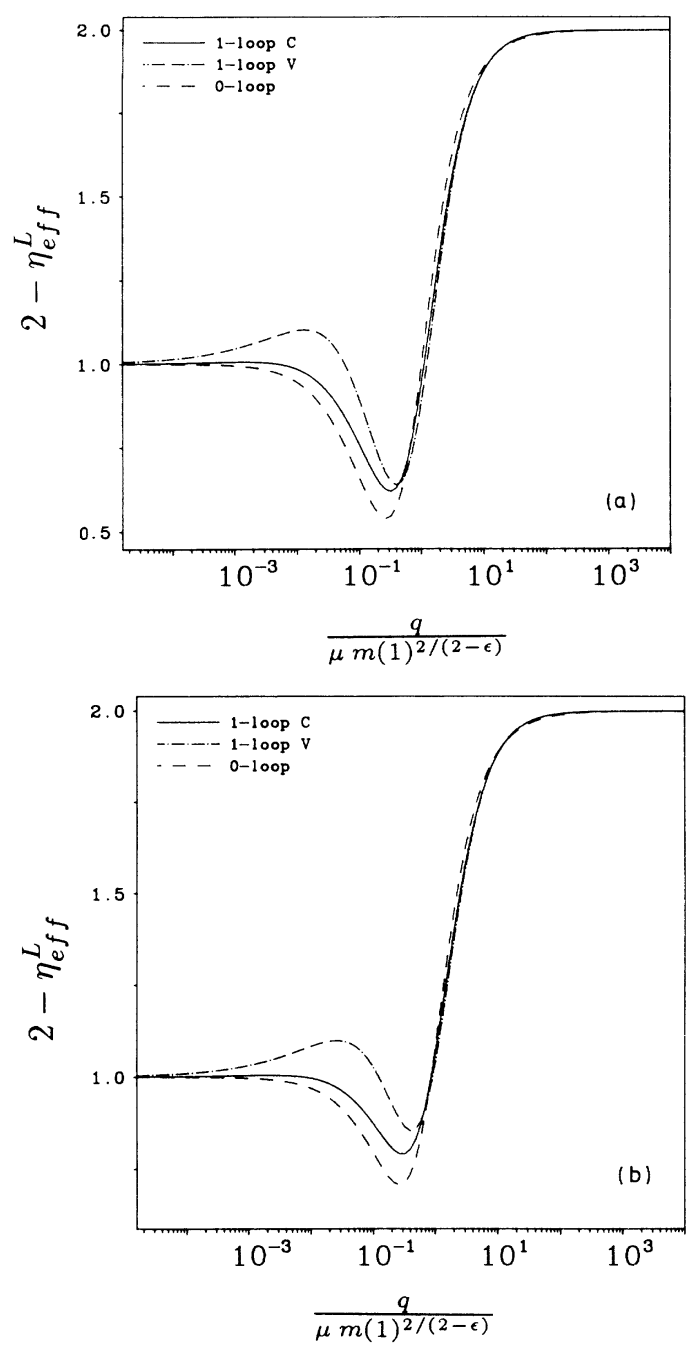

FIG. 7. Effective exponent $2-\eta_{\text {eff }}^{L}$ for $n=$ (a) 2 and (b) 3 .

which should be strictly discerned from the exponent $\eta=0$ obtained from the scaling relation $(5.3 \mathrm{~b})$. The effective exponent $2-\eta_{\text {eff }}^{L}$ as derived from the scaling function $\chi_{L}(\mathbf{q}, 0)$ is plotted in Fig. 7 for $n=2$ and 3. It displays a distinct minimum just before the scaling variable has reached the value 1 . Our interpretation for this possibly unexpected fact is the following. On leaving the critical region, the longitudinal mode "freezes out." However, the weight of the longitudinal fluctuation is considerably larger than that of a single transverse mode [there is a factor 9: $(n-1)$ in the diagrammatic contributions], and therefore the longitudinal response function at first behaves as in the case of a single-component order parameter. When the asymptotic limit is further approached, the Goldstone modes come into play and eventually prevail, leading to the coexistence anomalies. If this interpretation is correct, the minimum of the effective longitudinal exponent should become less pronounced if the number of components $n$ is increased, and this is precisely what happens [Fig. 7(b)]. In the spherical limit, the crossover is in fact perfectly smooth. ${ }^{24}$

\section{B. Dynamical correlation functions for model A}

With the same procedure as for the static properties, we now investigate the dynamical behavior, starting with the dissipative relaxation of model A $(a=0)$. For vanishing momentum, $\mathbf{q}=0$, the transverse loop exhibits an infrared singularity $\propto \omega^{-\epsilon / 2}$, which can be removed by using the matching condition

$$
l=\left(\frac{\omega}{\lambda \mu^{2}}\right)^{1 / 2}
$$

which again is a special case of (5.1). According to the dynamical scaling hypothesis ${ }^{32,33}$ and the definition of the dynamical critical exponent $z$ near $T_{c}$, the scaling variable obtained from (4.24b) must be proportional to

$$
\frac{l^{2}}{m(1)^{4 /(2-\epsilon)}} \propto \frac{\omega}{\lambda \mu^{2} \bar{\phi}^{4 /(2-\epsilon)}} \propto \frac{\omega}{|\tau|^{z v}},
$$

from which we conclude

$$
z=2 \text {. }
$$

Again, this coincides with the exact result in the critical regime $z=2+c \eta$ (Refs. 2 and 3) using $\eta=0$ to one-loop order. The dynamical response functions can now be obtained from the diagrams in Appendix B. For $q=0$ Feynman parametrization can be avoided and we find, for the cumulants $(C)$,

$$
\begin{aligned}
& \chi_{T}(0, \omega)=\frac{1}{\mu^{2} l^{2}}\left[i+\frac{u(l)}{3 \epsilon} m(l)^{2-\epsilon}\left\{1-\frac{m(l)^{\epsilon}}{-i+m(l)^{2}}\left[\frac{-i+m(l)^{2}}{2}\right\}^{1-\epsilon / 2}\right.\right. \\
& \left.\left.-\frac{m(l)^{\epsilon}}{i+m(l)^{2}}\left[m(l)^{2-\epsilon}-\left[\frac{-i+m(l)^{2}}{2}\right]^{1-\epsilon / 2}\right]\right\}\right]\left.\right|_{l=\left(\omega / \lambda \mu^{2}\right)^{1 / 2}}, \\
& \chi_{L}(0, \omega)=\left.\frac{1+\frac{i u(l) m(l)^{2}}{-i+m(l)^{2}}\left[\frac{n-1}{3 \epsilon}\left[\frac{-i}{2}\right]^{1-\epsilon / 2}-\frac{3}{\epsilon}\left[m(l)^{2-\epsilon}-\left[\frac{-i}{2}+m(l)^{2}\right]^{1-\epsilon / 2}\right]\right]}{\mu^{2} l^{2}\left[-i+m(l)^{2} Z_{m}(l)\right]}\right|_{l=\left(\omega / \lambda \mu^{2}\right)^{1 / 2}},
\end{aligned}
$$


where $0 \leq \epsilon<2$. Taking advantage of the fluctuationdissipation theorem $(2.21 \mathrm{~b})$, we can then calculate the dynamical correlation functions. The one-loop results for the two-point vertex functions $(V)$ are easily found by expanding with respect to $u$.

For $l \rightarrow 0$ the scaling functions behave as $\chi_{T}^{-1} \propto-i l^{2}$ and hence

$$
G_{T}(0, \omega) \propto \omega^{-2},
$$

and $\chi_{L}^{-1} \propto(2-i) l^{\epsilon}$; therefore

$$
\begin{aligned}
& \operatorname{Re} \chi_{L}(0, \omega) \propto \omega^{-\epsilon / 2}, \\
& G_{L}(0, \omega) \propto \omega^{-1-\epsilon / 2} .
\end{aligned}
$$

Again the "naive" singularity as read off from the transverse loop gives the correct behavior. The characteristic divergency $\propto \omega^{-\epsilon / 2}$ has been already found by Mazenko $^{14}$ and Schäfer. ${ }^{16}$ Figure 8 shows the logarithmic derivatives with respect to the frequency of the inverse real part of the longitudinal dynamical susceptibility [Fig. $8(\mathrm{a})]$ and the inverse longitudinal correlation function [Fig. 8(b)], respectively, for $n=2$ and $\epsilon=1$. The interpretation of the profound minimum in Figs. 8(a) and 8(b), whose depth decreases with larger $n$, is of course the same as for the static response function (see the preceding section).

Obviously, the behavior at the critical fixed point is preferably to be described by the vertex functions $(V)$, as can be seen in Fig. 8(a), where the tiny second dip in the curve for the cumulant $(C)$ is presumably an artifact of the one-loop theory. However, on approaching the coexistence limit, the one-loop cumulants will provide the correct results. The zero-loop contribution alone is not sufficient to yield the qualitative features of the imaginary part (compare Fig. 9 for model B below).

In the case of a nonconserved order parameter, the momentum- and frequency-dependent dynamical susceptibilities read, in three dimensions $(\epsilon=1)$,
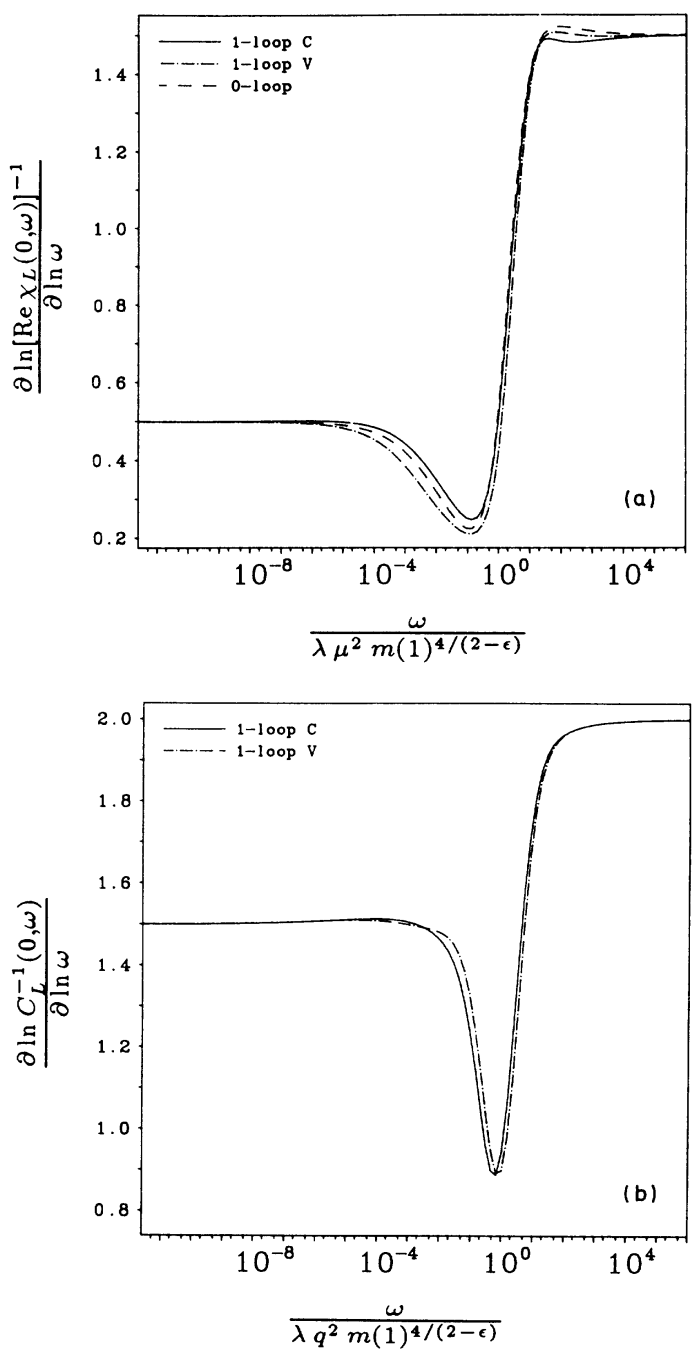

FIG. 8. Frequency dependence of (a) $\operatorname{Re} \chi_{L}(0, \omega)^{-1}$ and (b) $G_{L}(0, \omega)^{-1}$ for the relaxational model A; $\epsilon=1$ and $n=2$.

$$
\begin{aligned}
& \chi_{T}(\mathbf{q}, \omega)=\frac{1}{q^{2}-i \omega / \lambda}\left\{1-\frac{u(l) m(l) / 6}{\frac{q^{2}-i \omega / \lambda}{\mu^{2} l^{2}}}\left[2-\frac{m(l)}{q / \mu l} \mid \frac{\pi}{2}-\arcsin \frac{\frac{-i \omega}{\lambda \mu^{2} l^{2}}+m(l)^{2}}{\frac{q^{2}-i \omega / \lambda}{\mu^{2} l^{2}}+m(l)^{2}}\right.\right. \\
& +\arcsin \frac{\frac{i \omega}{\lambda \mu^{2} l^{2}}+m(l)^{2}}{\left[\left[\frac{q^{2}-i \omega / \lambda}{\mu^{2} l^{2}}-m(l)^{2}\right]^{2}+4 m(l)^{2} \frac{q^{2}}{\mu^{2} l^{2}}\right]^{1 / 2}} \\
& \left.\left.+\arcsin \frac{\frac{q^{2}-i \omega / \lambda}{\mu^{2} l^{2}}-m(l)^{2}}{\left.\left[\left[\frac{q^{2}-i \omega / \lambda}{\mu^{2} l^{2}}-m(l)^{2}\right]^{2}+4 m(l)^{2} \frac{q^{2}}{\mu^{2} l^{2}}\right]^{1 / 2}\right]}\right]\right\},
\end{aligned}
$$




$$
\begin{aligned}
& \chi_{L}(\mathbf{q}, \omega)=\frac{1}{q^{2}-i \omega / \lambda+\mu^{2} l^{2} m(l)^{2} Z_{m}(l)} \\
& \times\left\{1+\frac{1}{\frac{q^{2}-i \omega / \lambda}{\mu^{2} l^{2}}+m(l)^{2}} \frac{u(l) m(l)^{2}}{2 q / \mu l}\right. \\
& \times\left(\frac{n-1}{3}\left(\frac{\pi}{2}+\arcsin \frac{i \omega / \lambda}{q^{2}-i \omega / \lambda}\right)\right. \\
& +3 \int \arcsin \frac{\frac{i \omega}{\lambda \mu^{2} l^{2}}}{\left[\left[\frac{q^{2}-i \omega / \lambda}{\mu^{2} l^{2}}\right]^{2}+4 m(l)^{2} \frac{q^{2}}{\mu^{2} l^{2}}\right]^{1 / 2}} \\
& \left.\left.+\arcsin \frac{\frac{q^{2}-i \omega / \lambda}{\mu^{2} l^{2}}}{\left[\left[\frac{q^{2}-i \omega / \lambda}{\mu^{2} l^{2}}\right]^{2}+4 m(l)^{2} \frac{q^{2}}{\mu^{2} l^{2}}\right]^{1 / 2}}\right] \mid\right) .
\end{aligned}
$$

By investigation of the poles of Eqs. (5.13), we can determine the dispersion relations of the asymptotically prevailing excitations. For the Goldstone modes we derive, from (5.13a),

$$
i \omega(\mathbf{q})=\lambda q^{2}
$$

i.e., diffusive relaxation, as expected.

Turning to the longitudinal response function, we note that the pole (5.14) is reflected in $(5.13 \mathrm{~b})$ as a logarithmic singularity, for

$$
\arcsin \frac{i \omega}{z}=-i \ln \frac{\left(z^{2}+\omega^{2}\right)^{1 / 2}-\omega}{z} \rightarrow-i \ln \frac{z}{2 \omega},
$$

as $z \rightarrow 0$. Therefore it can be eliminated if, as in (5.1), the absolute value of the inverse transverse propagator is identified with the square of the flow parameter:

$$
l^{2}=\left|\frac{q^{2}}{\mu^{2}}-\frac{i \omega}{\lambda \mu^{2}}\right| \text {. }
$$

By insertion of (5.15) into (5.13b), one finds that (5.14) leads to the algebraic singularity

$$
\chi_{L}(\mathrm{q}, \omega) \propto\left|\frac{q^{2}}{\mu^{2}}-\frac{i \omega}{\lambda \mu^{2}}\right|^{-\epsilon / 2} .
$$

We remark that on approaching the branch cut one has to use the familiar replacement $\omega \rightarrow \omega+i \eta, \eta \downarrow 0$, for the retarded Green function. Although the longitudinal fluctuations have died out, there is a divergency of the longitudinal susceptibility caused by the resonant manifestation of the massless transverse modes (compare the corresponding results of the $1 / n$ expansion $\left.{ }^{24}\right)$. This is reflected in the fact that asymptotically only the transverse timescale $\lambda_{1}$, which enters the general matching condition (5.1), appears in the physical quantities, while the parameter $\lambda_{\|}$drops out of the theory. The exact result (5.16) for the longitudinal susceptibility in the coexistence limit implies a very anomalous line shape in comparison to the Lorentzian form of the transverse correlation function. This should lead to a pronounced effect with regard to the long-time behavior of the dynamical response functions $\chi_{L}(\mathbf{x}, t)$ or $\chi_{L}(\mathbf{q}, t)$, respectively.

\section{Dynamical correlation functions for model B}

In the case of a conserved order parameter, model $\mathbf{B}$ with $a=2$, we cannot simply set $\mathrm{q}=0$ because of the diffusion pole. Rather, the ratio $\omega / \lambda q^{2}$ is to be kept fixed and then the limit $\mathbf{q} \rightarrow 0$ may be considered. According to $(5.1)$, the matching condition hence becomes

$$
l=\left[\frac{\omega}{\lambda q^{2}}\right]^{1 / 2}
$$

from which, by noting

$$
\begin{aligned}
\frac{l^{2}}{m(1)^{4 /(2-\epsilon)}} & \propto \frac{\omega}{\lambda(q \xi)^{2} \xi^{-2} \bar{\phi}^{4 /(2-\epsilon)}} \\
& \propto \frac{\omega}{(q \xi)^{2}|\tau|^{z v}},
\end{aligned}
$$

we find, for the dynamical critical exponent,

$$
z=4 \text {. }
$$

Using the relation (4.10), the general result is easily seen to be $z=4-\eta .^{4}$ 
In the limit $q \rightarrow 0$ with fixed scaling variable $\propto \omega / q^{2}$, the loop contributions to the dynamic susceptibilities vanish (see Appendix B) or are canceled by the renormalization constant $(4.11 \mathrm{c})$. Thus we have

$$
\begin{aligned}
& \chi_{T}^{-1}\left(0, \omega / q^{2}\right)=-\left.i \mu^{2} l^{2}\right|_{l=\left(\omega / \lambda q^{2}\right)^{1 / 2},} \\
& \chi_{L}^{-1}\left(0, \omega / q^{2}\right)=\left.\mu l^{2}\left[-i+m(l)^{2}\right]\right|_{l=\left(\omega / \lambda q^{2}\right)^{1 / 2}},
\end{aligned}
$$

leading to the asymptotic behavior

$$
\begin{aligned}
& G_{T}\left(0, \omega / q^{2}\right) \propto\left(\omega / q^{2}\right)^{-2}, \\
& \operatorname{Re} \chi_{L}\left(0, \omega / q^{2}\right) \propto\left(\omega / q^{2}\right)^{-\epsilon / 2}, \\
& G_{L}\left(0, \omega / q^{2}\right) \propto\left(\omega / q^{2}\right)^{-\epsilon} .
\end{aligned}
$$

In Fig. 9 the logarithmic derivatives of (5.19b) with respect to the scaling variable are shown [compare with the analogous plot for model A (Fig. 7)]. Note that the imaginary part of the susceptibility and therefore the dynamical correlation function obey different power laws than in the case of a nonconserved order parameter in the coexistence limit.
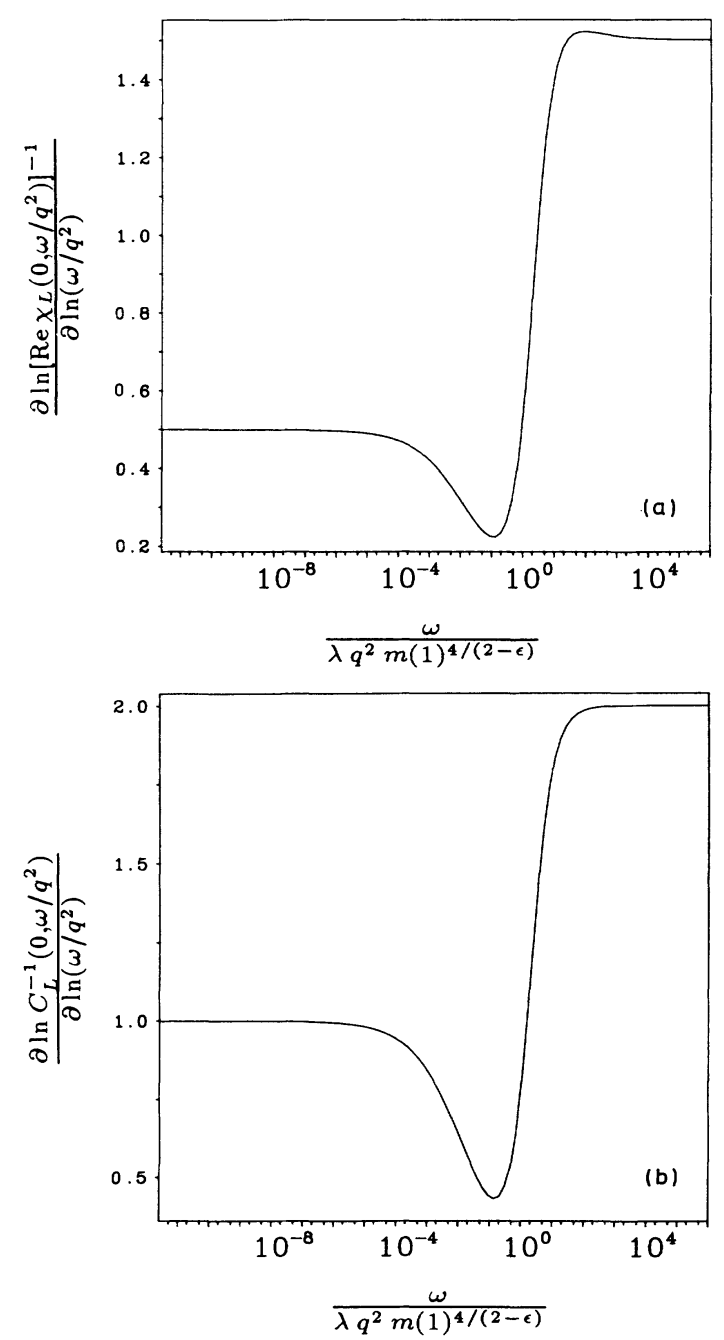

FIG. 9. Frequency dependence of (a) $\operatorname{Re} \chi_{L}\left(0, \omega / q^{2}\right)^{-1}$ and (b) $G_{L}\left(0, \omega / q^{2}\right)^{-1}$ for the relaxational model B; $\epsilon=1$ and $n=2$. Compare Fig. 8.
Without introducing flow-dependent parameters as $m(l)$ via the renormalization-group equation, from the zero-loop term (5.15b) no coexistence anomalies can be deduced. ${ }^{14}$ For the special situation $q=0$, instead of (5.20b), Schäfer claimed an $\omega^{-\epsilon / 4}$ singularity of the longitudinal response function. ${ }^{16}$ This does not incorporate the relevant scaling variable itself, and with such an exponentiation the well-established feature (2.1) cannot be reproduced. The canonical behavior of the transverse fields alone does not permit an omission of the renormalization-group techniques, because the anomalous dimension of the mass parameter $(4.23 \mathrm{~b})$ still has to be taken into account. The discrepancy as compared to a $1 / n$ expansion may possibly be sought in the fact that a model with diffusive dynamics is ill defined in the spherical limit $n \rightarrow \infty$. This is because the hydrodynamic limit is confined to frequencies lower than a typical collision rate, which is of the order $1 / n .{ }^{34,35}$

The dispersion of both the transverse and longitudinal modes in the coexistence limit is described by

$$
i \omega(\mathbf{q})=\lambda q^{4},
$$

employing the matching condition [see Eq. (5.1)]

$$
l^{2}=\left|\frac{q^{2}}{\mu^{2}}-\frac{i \omega}{\lambda q^{2}}\right| .
$$

The singularity of the longitudinal response function appears as

$$
\chi_{L}(\mathbf{q}, \omega) \propto\left|\frac{q^{2}}{\mu^{2}}-\frac{i \omega}{\lambda q^{2}}\right|^{-\epsilon / 2},
$$

where again $\omega \rightarrow \omega+i \eta, \eta \downarrow 0$, should be inserted in order to avoid ambiguities.

Both for conserved and nonconserved order parameters, the dynamical susceptibilities display the general scaling law la $^{32,33}$

$$
\chi_{T / L}^{-1}(\tau, \mathbf{q}, \omega)=q^{2-\eta} \hat{\chi}_{T / L}^{-1}\left(q \xi, \omega /|\tau|^{z v}\right) .
$$

Remarkably, the leading asymptotic expressions for the longitudinal and transverse response functions in the coexistence limit are related through

$$
\chi_{L}(\mathbf{q}, \omega)^{-1} \propto\left|\chi_{T}(\mathbf{q}, \omega)^{-1}\right| \epsilon / 2 ;
$$

a similar formula has been obtained for the static case by Brézin and Wallace. ${ }^{7}$

\section{SUMMARY AND OUTLOOK}

We have investigated the dynamical critical behavior of the isotropic relaxational models $A$ and $B$ with nonconserved and conserved order parameters, respectively, in the phase with broken continuous global symmetry. On the basis of the path-integral formulation of dynamical perturbation theory according to Bausch, Janssen, Wagner $^{4}$ and De Dominicis, ${ }^{5}$ a method has been described to treat both the critical and Goldstone singularities within a generalized regularization scheme proposed by Amit and Goldschmidt. ${ }^{18}$ A similar study of the static properties of the $\phi^{4}$ model below the transition temperature by Lawrie ${ }^{19,20}$ was extended in two respects. First, 
the $\epsilon$ expansion was avoided, arguing that the asymptotic theory is exactly treatable, and hence the perturbation series for the cumulants reduces to the one-loop contributions. This corresponds to the fact that in the coexistence limit the leading order of the $1 / n$ expansion correctly yields the two-point vertex functions. Second, the full flow equations were solved numerically, which allows a more detailed study of the crossover between the isotropic Heisenberg fixed point (4.22) and the coexistence fixed point (4.23). Especially, the scaling behavior with (4.25) should be emphasized, through which the scaling variables of the static and dynamic theory can be defined.

Generally, the singularities caused by the Goldstone modes are characterized by the anomalous dimension of the mass parameter $\zeta_{m C}^{*}=-2+\epsilon$. At the upper critical dimension $d_{c}=4$, they are reduced to mere logarithmic corrections, while in two dimensions the assumption of a spontaneously broken symmetry with homogeneous order parameter proves to be inconsistent, in accordance with the Mermin-Wagner theorem. ${ }^{27,28}$ Therefore the threedimensional case is the really interesting one with respect to coexistence anomalies.

As an application of the general theory, the static and dynamical response functions have been calculated. The expressions (5.5) for the static susceptibilities, and (5.11) and (5.13) for the purely dissipative dynamics of model

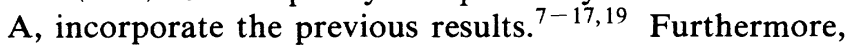
they predict pronounced minima of the logarithmic derivatives of the longitudinal scaling functions, to be interpreted as the temporary dominance of the massive excitation, while eventually the Goldstone modes prevail. We also emphasize again the anomalous longitudinal line shape [see Eq. (5.16)]. These striking features might possibly be experimentally observed (see our remarks below). The case of model B with conserved order parameter seems to be clarified. Our results for the scaling functions (5.19) display the correct scaling behavior, which is based on the nontrivial property (4.24b), and the asymptotic transverse and longitudinal susceptibilities are related through (5.25). The power laws describing the coexistence anomalies are summarized in Table $I$.

The investigations of the relaxational models, on the one hand, serves to elaborate the theory and test the formalism, as they describe most of the real systems only very crudely, such as superfluid helium 4 (model $A$ with $n=2$ ), isotropic ferromagnets (model B with $n=3$ ), or isotropic antiferromagnets (model $\mathrm{A}$ with $n=3$ ), because mode-coupling terms are omitted. In the case of superfluid helium 4, for example, this means that the
TABLE I. Coexistence anomalies.

\begin{tabular}{ccc}
\hline \hline & Model A & Model B \\
\hline$\chi_{L}(\mathbf{q}, 0)$ & $\propto q^{-\epsilon}$ & $\propto q^{-\epsilon}$ \\
$\operatorname{Re} \chi_{L}\left(0, \omega / q^{a}\right)$ & $\propto \omega^{-\epsilon / 2}$ & $\propto\left(\omega / q^{2}\right)^{-\epsilon / 2}$ \\
$G_{L}\left(0, \omega / q^{a}\right)$ & $\propto \omega^{-1-\epsilon / 2}$ & $\propto\left(\omega / q^{2}\right)^{-\epsilon}$ \\
\hline \hline
\end{tabular}

propagating character of second sound is neglected. On the other hand, however, there is also an important application of our theory to real systems, such as the crystals with general structure $A_{2} B X_{4}$ displaying a commensurate-to-incommensurate phase transition, which fall into the universality class of the isotropic relaxational model A. Unfortunately, precise measurements of the response functions are very difficult to achieve and have not yet been reported. However, the characteristic minima in Figs. 7-9 might be detectable, and also the effect of the anomalous line shape could be observed, especially when probing the long-time limit. Model B, on the other hand, should be regarded as more artificial, for in $\mathrm{O}(n)$-symmetrical systems with conserved order parameter (e.g., Heisenberg ferromagnets), modecoupling terms are relevant.

One should also be aware of the fact that in the ideally $\mathrm{O}(2)$-symmetric helium 4 there is no physical field conjugate to the order parameter, and hence the longitudinal susceptibility cannot be measured. However, there are other physical quantities showing coexistence anomalies, e.g., the frequency-dependent specific heat which constitutes the sound-absorption coefficient. The application of an extended version of our theory indeed describes the experimental situation satisfactorily, ${ }^{23}$ which may elucidate the power of the presented technique.

For many real systems, such as superfluid helium 4 or magnets, of course one has to take anisotropies and/or mode-coupling vertices into account. One of the major advantages of Lawrie's scheme is that it produces results of fairly transparent structure. Therefore we expect that even more complicated models can be investigated, which has not yet been possible with any of the former methods. We plan to report on some results in a forthcoming communication.

\section{ACKNOWLEDGMENT}

This work has been supported by the Deutsche Forschungsgemeinschaft (DFG) under Contract No. Schw. 348/4-1.

\section{APPENDIX A: WARD-TAKAHASHI IDENTITIES}

By performing suitable functional derivatives on the general Ward-Takahashi identity (3.4b), a number of important exact relations between vertex functions of different order can be derived. They are valid both in the ordered and nonordered phases. Including the four-point vertex functions, the relevant results read

$$
\begin{aligned}
& \bar{\phi} \Gamma_{\tilde{\pi}^{\alpha} \pi^{\beta} \sigma}(-\underline{k} ; 0 ; \underline{k})=\bar{\phi} \Gamma_{\tilde{\pi} \pi \sigma}(-\underline{k} ; 0 ; \underline{k}) \delta^{\alpha \beta}=\left[\Gamma_{\tilde{\sigma} \sigma}(\underline{k})-\Gamma_{\tilde{\pi} \pi}(\underline{k})\right] \delta^{\alpha \beta}, \\
& \bar{\phi} \Gamma_{\tilde{\sigma} \pi^{\alpha} \pi^{\beta}}(-\underline{k} ; 0 ; \underline{k})=\bar{\phi} \Gamma_{\tilde{\sigma} \pi \pi}(-\underline{k} ; 0 ; \underline{k}) \delta^{\alpha \beta}=\left[\Gamma_{\tilde{\sigma} \sigma}(\underline{k})-\Gamma_{\tilde{\pi} \pi}(\underline{k})\right] \delta^{\alpha \beta}, \\
& \bar{\phi} \Gamma_{\tilde{\pi}^{\alpha} \tilde{\sigma} \pi^{\beta}}(-\underline{k} ; \underline{k} ; 0)=\bar{\phi} \Gamma_{\tilde{\pi} \tilde{\sigma} \pi}(-\underline{k} ; \underline{k} ; 0) \delta^{\alpha \beta}=\left[\Gamma_{\tilde{\sigma} \tilde{\sigma}}(\underline{k})-\Gamma_{\tilde{\pi} \tilde{\pi}}(\underline{k})\right] \delta^{\alpha \beta},
\end{aligned}
$$




$$
\begin{aligned}
& \bar{\phi} \Gamma_{\tilde{\pi}^{\alpha} \pi^{\beta} \pi^{\gamma} \pi^{\delta}}(-\underline{k} ; 0 ; \underline{k} / 2 ; \underline{k} / 2)=\bar{\phi}_{0} \Gamma_{\tilde{\pi} \pi \pi \pi}(-\underline{k} ; 0 ; \underline{k} / 2 ; \underline{k} / 2) F^{\alpha \beta \gamma \delta}=\left[\Gamma_{\tilde{\sigma} \pi \pi}(-\underline{k} ; \underline{k} / 2 ; \underline{k} / 2)+2 \Gamma_{\tilde{\pi} \pi \sigma}(-\underline{k} ; \underline{k} / 2 ; \underline{k} / 2)\right] F^{\alpha \beta \gamma \delta}, \\
& \bar{\phi} \Gamma_{\tilde{\pi}^{\alpha} \pi^{\beta} \sigma \sigma}(-\underline{k} ; 0 ; \underline{k} / 2 ; \underline{k} / 2)=\bar{\phi} \Gamma_{\tilde{\pi} \pi \sigma \sigma}(-\underline{k} ; 0 ; \underline{k} / 2 ; \underline{k} / 2) \delta^{\alpha \beta}=\left[\Gamma_{\tilde{\sigma} \sigma \sigma}(-\underline{k} ; \underline{k} / 2 ; \underline{k} / 2)-2 \Gamma_{\tilde{\pi} \pi \sigma}(-\underline{k} ; \underline{k} / 2 ; \underline{k} / 2)\right] \delta^{\alpha \beta}, \\
& \bar{\phi} \Gamma_{\tilde{\sigma} \pi^{\alpha} \pi^{\beta} \sigma^{\beta}}(-\underline{k} ; 0 ; \underline{k} / 2 ; \underline{k} / 2)=\bar{\phi} \Gamma_{\tilde{\sigma} \pi \pi \sigma}(-\underline{k} ; 0 ; \underline{k} / 2 ; \underline{k} / 2) \delta^{\alpha \beta} \\
& =\left[\Gamma_{\tilde{\sigma} \sigma \sigma}(-\underline{k} ; \underline{k} / 2 ; \underline{k} / 2)-\Gamma_{\tilde{\sigma} \pi \pi}(-\underline{k} ; \underline{k} / 2 ; \underline{k} / 2)-\Gamma_{\tilde{\pi} \pi \sigma}(-\underline{k} ; \underline{k} / 2 ; \underline{k} / 2)\right] \delta^{\alpha \beta}, \\
& \bar{\phi} \Gamma_{\tilde{\pi}^{\alpha} \pi^{\beta} \pi^{\gamma} \pi^{\delta} \sigma}(-\underline{k} ; 0 ; \underline{k} / 3 ; \underline{k} / 3 ; \underline{k} / 3)=\bar{\phi} \Gamma_{\tilde{\pi} \pi \pi \pi \sigma}(-\underline{k} ; 0 ; \underline{k} / 3 ; \underline{k} / 3 ; \underline{k} / 3) F^{\alpha \beta \gamma \delta} \\
& =\left[\Gamma_{\tilde{\sigma} \pi \pi \sigma}(-\underline{k} ; \underline{k} / 3 ; \underline{k} / 3 ; \underline{k} / 3)+2 \Gamma_{\tilde{\pi} \pi \sigma \sigma}(-\underline{k} ; \underline{k} / 3 ; \underline{k} / 3 ; \underline{k} / 3)\right. \\
& \left.-\Gamma_{\tilde{\pi} \pi \pi \pi}(-\underline{k} ; \underline{k} / 3 ; \underline{k} / 3 ; \underline{k} / 3)\right] F^{\alpha \beta \gamma \delta}, \\
& \bar{\phi} \Gamma_{\tilde{\pi}^{\alpha} \pi^{\beta} \sigma \sigma \sigma}(-\underline{k} ; 0 ; \underline{k} / 3 ; \underline{k} / 3 ; \underline{k} / 3)=\bar{\phi} \Gamma_{\tilde{\pi} \pi \sigma \sigma \sigma}(-\underline{k} ; 0 ; \underline{k} / 3 ; \underline{k} / 3 ; \underline{k} / 3) \delta^{\alpha \beta} \\
& =\left[\Gamma_{\tilde{\sigma} \sigma \sigma \sigma}(-\underline{k} ; \underline{k} / 3 ; \underline{k} / 3 ; \underline{k} / 3)-3 \Gamma_{\tilde{\pi} \pi \sigma \sigma}(-\underline{k} ; \underline{k} / 3 ; \underline{k} / 3 ; \underline{k} / 3)\right] \delta^{\alpha \beta}, \\
& \bar{\phi} \Gamma_{\tilde{\sigma} \pi^{\alpha} \pi^{\beta} \pi^{\gamma} \pi^{\delta}}(-\underline{k} ; 0 ; \underline{k} / 3 ; \underline{k} / 3 ; \underline{k} / 3)=\bar{\phi} \Gamma_{\tilde{\sigma} \pi \pi \pi \pi}(-\underline{k} ; 0 ; \underline{k} / 3 ; \underline{k} / 3 ; \underline{k} / 3) F^{\alpha \beta \gamma \delta} \\
& =\left[3 \Gamma_{\tilde{\sigma} \pi \pi \sigma}(-\underline{k} ; \underline{k} / 3 ; \underline{k} / 3 ; \underline{k} / 3)-\Gamma_{\tilde{\pi} \pi \pi \pi}(-\underline{k} ; \underline{k} / 3 ; \underline{k} / 3 ; \underline{k} / 3)\right] F^{\alpha \beta \gamma \delta}, \\
& \bar{\phi} \Gamma_{\tilde{\sigma} \pi^{\alpha} \pi^{\beta} \sigma \sigma}(-\underline{k} ; 0 ; \underline{k} / 3 ; \underline{k} / 3 ; \underline{k} / 3)=\bar{\phi} \Gamma_{\tilde{\sigma} \pi \pi \sigma \sigma}(-\underline{k} ; 0 ; \underline{k} / 3 ; \underline{k} / 3 ; \underline{k} / 3) \delta^{\alpha \beta} \\
& =\left[\Gamma_{\tilde{\sigma} \sigma \sigma \sigma}(-\underline{k} ; \underline{k} / 3 ; \underline{k} / 3 ; \underline{k} / 3)-2 \Gamma_{\tilde{\sigma} \pi \pi \sigma}(-\underline{k} ; \underline{k} / 3 ; \underline{k} / 3 ; \underline{k} / 3)\right. \\
& \left.-\Gamma_{\tilde{\pi} \pi \sigma \sigma}(-\underline{k} ; \underline{k} / 3 ; \underline{k} / 3 ; \underline{k} / 3)\right] \delta^{\alpha \beta} \text {, }
\end{aligned}
$$

where the abbreviation $\underline{k}=(\mathbf{q}, \omega)$ has been introduced.

\section{APPENDIX B: TWO-POINT VERTEX FUNCTIONS}

In this appendix we list the zero- and one-loop diagrams (Fig. 10) and the corresponding analytical results for the two-point vertex functions of the time-dependent Ginzburg-Landau models $\mathrm{A}(a=0)$ and $\mathrm{B}(a=2)$. The integration over internal frequencies has already been performed by means of the residue theorem.

(a)

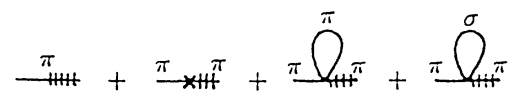

(b)

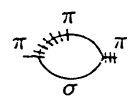

(c)

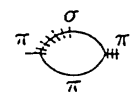

(d)

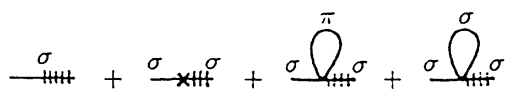

(e)

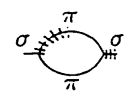

(f)

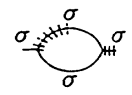

(g)<smiles>C[14CH3]</smiles>

(h)

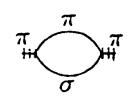

(i)

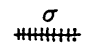

(j)

(k)
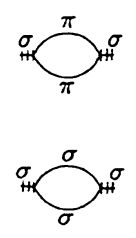

FIG. 10. Zero- and one-loop diagrams for the two-point vertex functions of models $A(a=0)$ and $B(a=2)$. The corresponding explicit analytical expressions are listed in Appendix B. 
$\Gamma_{0 \tilde{\pi} \pi}(\mathbf{q}, \omega):$

$$
\begin{aligned}
& \text { (a) }=i \omega+\lambda_{0} q^{a}\left(q^{2}+\frac{1}{3} u_{0} m_{0}^{2} \int_{k} \frac{1}{k^{2}\left(m_{0}^{2}+k^{2}\right)}\right) \\
& \text { (b) }=-\lambda_{0} q^{a} \frac{1}{3} u_{0} m_{0}^{2} \int_{k} \frac{(\mathbf{q} / 2+\mathbf{k})^{a}}{m_{0}^{2}+(\mathbf{q} / 2-\mathbf{k})^{2}} \frac{1}{i \omega / \lambda_{0}+(\mathbf{q} / 2+\mathbf{k})^{2+a}+(\mathbf{q} / 2-\mathbf{k})^{a}\left[m_{0}^{2}+(\mathbf{q} / 2-\mathbf{k})^{2}\right]} \\
& \text { (c) }=-\lambda_{0} q^{a} \frac{1}{3} u_{0} m_{0}^{2} \int_{k} \frac{(\mathbf{q} / 2+\mathbf{k})^{a}}{(\mathbf{q} / 2-\mathbf{k})^{2}} \frac{1}{i \omega / \lambda_{0}+(\mathbf{q} / 2+\mathbf{k})^{a}\left[m_{0}^{2}+(\mathbf{q} / 2+\mathbf{k})^{2}\right]+(\mathbf{q} / 2-\mathbf{k})^{2+a}}
\end{aligned}
$$

$\Gamma_{0 \tilde{\sigma} \sigma}(\mathbf{q}, \omega):$

$$
\begin{aligned}
& \text { (d) }=i \omega+\lambda_{0} q^{a}\left(m_{0}^{2}+q^{2}\right) \\
& \text { (e) }=-\lambda_{0} q^{a} \frac{n-1}{3} u_{0} m_{0}^{2} \int_{k} \frac{(\mathbf{q} / 2+\mathbf{k})^{a}}{(\mathbf{q} / 2-\mathbf{k})^{2}} \frac{1}{i \omega / \lambda_{0}+(\mathbf{q} / 2+\mathbf{k})^{2+a}+(\mathbf{q} / 2-\mathbf{k})^{2+a}} \\
& \text { (f) }=-\lambda_{0} q^{a} 3 u_{0} m_{0}^{2} \int_{k} \frac{(\mathbf{q} / 2+\mathbf{k})^{a}}{m_{0}^{2}+(\mathbf{q} / 2-\mathbf{k})^{2}} \frac{1}{i \omega / \lambda_{0}+(\mathbf{q} / 2+\mathbf{k})^{a}\left[m_{0}^{2}+(\mathbf{q} / 2+\mathbf{k})^{2}\right]+(\mathbf{q} / 2-\mathbf{k})^{a}\left[m_{0}^{2}+(\mathbf{q} / 2-\mathbf{k})^{2}\right]} ;
\end{aligned}
$$

$\Gamma_{0 \tilde{\pi} \tilde{\pi}}(\mathbf{q}, \omega):$

$$
\begin{aligned}
& (\mathrm{g})=-2 \lambda_{0} q^{a} \\
& (\mathrm{~h})=-2 \lambda_{0} q^{2 a} \frac{1}{3} u_{0} m_{0}^{2} \operatorname{Re} \int_{k} \frac{1}{(\mathbf{q} / 2+\mathbf{k})^{2}\left[m_{0}^{2}+(\mathbf{q} / 2-\mathbf{k})^{2}\right]} \frac{1}{i \omega / \lambda_{0}+(\mathbf{q} / 2+\mathbf{k})^{2+a}+(\mathbf{q} / 2-\mathbf{k})^{a}\left[m_{0}^{2}+(\mathbf{q} / 2-\mathbf{k})^{2}\right]}
\end{aligned}
$$

$\Gamma_{0 \tilde{\sigma} \tilde{\sigma}}(\mathbf{q}, \omega):$

$$
\begin{aligned}
& \text { (i) }=-2 \lambda_{0} q^{a} \\
& \text { (j) }=-2 \lambda_{0} q^{2 a} \frac{n-1}{6} u_{0} m_{0}^{2} \operatorname{Re} \int_{k} \frac{1}{(\mathbf{q} / 2+\mathbf{k})^{2}(\mathbf{q} / 2-\mathbf{k})^{2}} \frac{1}{i \omega / \lambda_{0}+(\mathbf{q} / 2+\mathbf{k})^{2+a}+(\mathbf{q} / 2-\mathbf{k})^{2+a}} \\
& (\mathrm{k})=-2 \lambda_{0} q^{2 a} \frac{3}{2} u_{0} m_{0}^{2} \operatorname{Re} \int_{k} \frac{1}{\left[m_{0}^{2}+(\mathbf{q} / 2+\mathbf{k})^{2}\right]\left[m_{0}^{2}+(\mathbf{q} / 2-\mathbf{k})^{2}\right]} \\
& \times \frac{1}{i \omega / \lambda_{0}+(\mathbf{q} / 2+\mathbf{k})^{a}\left[m_{0}^{2}+(\mathbf{q} / 2+\mathbf{k})^{2}\right]+(\mathbf{q} / 2-\mathbf{k})^{a}\left[m_{0}^{2}+(\mathbf{q} / 2-\mathbf{k})^{2}\right]} .
\end{aligned}
$$

${ }^{1}$ P. C. Hohenberg and B. I. Halperin, Rev. Mod. Phys. 49, 435 (1977).

${ }^{2}$ B. I. Halperin, P. C. Hohenberg, and S. Ma, Phys. Rev. Lett. 29, 1548 (1972); Phys. Rev. B 10, 139 (1974); 13, 4119 (1976).

${ }^{3}$ C. De Dominicis, E. Brézin, and J. Zinn-Justin, Phys. Rev. B 12, 4945 (1975); E. Brézin and C. De Dominicis, ibid. 12, 4954 (1975).

${ }^{4}$ H. K. Janssen, Z. Phys. B 23, 377 (1976); R. Bausch, H. K. Janssen, and H. Wagner, ibid. 24, 113 (1976).

${ }^{5}$ C. De Dominicis, J. Phys. (Paris) Colloq. 37, C1-247 (1976).

${ }^{6} \mathrm{~J}$. Goldstone, Nuovo Cimento 19, 154 (1961).

${ }^{7}$ E. Brézin and D. J. Wallace, Phys. Rev. B 7, 1967 (1973).

${ }^{8}$ T. Holstein and H. Primakoff, Phys. Rev. 58, 1098 (1940).

${ }^{9}$ F. Schwabl and K. H. Michel, Phys. Rev. B 2, 189 (1970).

${ }^{10}$ F. J. Dyson, Phys. Rev. 102, 1217 (1956); 102, 1230 (1956).

${ }^{11}$ D. J. Wallace and R. K. P. Zia, Phys. Rev. B 12, 5340 (1975).

${ }^{12}$ D. R. Nelson, Phys. Rev. B 13, 2222 (1976).

${ }^{13}$ E. Brézin and J. Zinn-Justin, Phys. Rev. B 14, 3110 (1976).

${ }^{14}$ G. F. Mazenko, Phys. Rev. B 14, 3933 (1976).
${ }^{15}$ L. Schäfer and H. Horner, Z. Phys. B 29, 251 (1978).

${ }^{16}$ L. Schäfer, Z. Phys. B 31, 289 (1978).

${ }^{17}$ J. F. Nicoll and T. S. Chang, Phys. Rev. A 17, 2083 (1978); J. F. Nicoll, Phys. Rev. B 21, 1124 (1980).

${ }^{18}$ D. J. Amit and Y. Y. Goldschmidt, Ann. Phys. (N.Y.) 114, 356 (1978).

${ }^{19}$ I. D. Lawrie, J. Phys. A 14, 2489 (1981).

${ }^{20}$ I. D. Lawrie, J. Phys. A 18, 1141 (1985).

${ }^{21} \mathrm{R}$. Schloms, dissertation thesis, Rheinisch-Westfälisch Technische Hochschule Aachen, 1989; R. Schloms and V. Dohm, Nucl. Phys. B328, 639 (1989); Phys. Rev. B 42, 6142 (1990).

${ }^{22}$ A. Schorgg and F. Schwabl (unpublished).

${ }^{23}$ A. Schorgg, diploma thesis, Technische Universität München, 1988; A. Schorgg and F. Schwabl, Phys. Rev. B (to be published).

${ }^{24}$ D. J. Amit, Field Theory, the Renormalization Group, and Critical Phenomena, 2nd ed. (World Scientific, Singapore, 1984).

${ }^{25}$ G. t' Hooft and M. Veltman, Nucl. Phys. B44, 189 (1972). 
${ }^{26}$ I. D. Lawrie, J. Phys. A 9, 961 (1976).

${ }^{27}$ N. D. Mermin and H. Wagner, Phys. Rev. Lett. 17, 1133 (1966).

${ }^{28}$ P. C. Hohenberg, Phys. Rev. 158, 383 (1967).

${ }^{29}$ K. Symanzik, Lett. Nuovo Cimento 8, 771 (1973).

${ }^{30} \mathbf{R}$. Dengler, dissertation thesis, Technische Universität München, 1987.

${ }^{31}$ E. Frey, dissertation thesis, Technische Universität München, 1989; E. Frey and F. Schwabl, J. Phys. (Paris) Colloq. 48, C8-
1569 (1988); Phys. Rev. B 42, 8261 (1990); 43, 833 (1991).

${ }^{32}$ R. A. Ferrell, N. Menyhárd, H. Schmidt, F. Schwabl, and P. Szépfalusy, Phys. Rev. Lett. 18, 891 (1967); Ann. Phys. (N.Y.) 47, 565 (1968).

${ }^{33}$ B. I. Halperin and P. C. Hohenberg, Phys. Rev. 177, 952 (1969).

${ }^{34}$ S. Ma and L. Senbetu, Phys. Rev. A 10, 2401 (1974).

${ }^{35}$ L. Sasvári, F. Schwabl, and P. Szépfalusy, Physica 81A, 108 (1975). 